\title{
On the achievable rates of a secondary link coexisting with a primary multiple access network
}

\author{
John Tadrous ${ }^{1 *}$ and Mohammed Nafie ${ }^{2,3}$
}

\begin{abstract}
An achievable rate region for a primary multiple access network coexisting with a secondary link of one transmitter and a corresponding receiver is analyzed. The rate region depicts the sum primary rate versus the secondary rate and is established assuming that the secondary link performs rate splitting. The achievable rate region is the union of two types of rate regions. The first type is a rate region established assuming that the secondary receiver cannot decode any primary signal, whereas the second is established assuming that the secondary receiver can decode the signal of one primary link. The achievable rate region is determined first assuming discrete memoryless channel (DMC), then the results are applied to a Gaussian channel. In the Gaussian channel, the performance of rate splitting is characterized for the two types of rate regions. Moreover, a necessary and sufficient condition to determine which primary signal the secondary receiver can decode without degrading the range of primary achievable sum rates is provided. When this condition is satisfied by a certain primary user, the secondary receiver can decode its signal and achieve larger rates without reducing the sum of the primary achievable rates as compared to the case in which it does not decode any primary signal. It is also shown that the probability of having at least one primary user satisfying this condition grows with the primary signal-to-noise ratio.
\end{abstract}

Keywords: Rate splitting; Cognitive radios; Discrete memoryless channels

\section{Introduction}

A potential benefit of allowing secondary users to share primary bands is the enhancement of the spectrum utilization. As introduced in [1,2], cognitive radios, or secondary users, are frequency-agile devices that can utilize unused spectrum bands through dynamic spectrum access. In dynamic spectrum access, secondary users should sense the spectrum and identify unused bands or spectrum holes. If a band is sensed and found to be in low use by primary users, i.e., underutilized, a secondary user may opportunistically access this band by adjusting its transmit parameters to fully utilize this band without causing excessive interference on the primary users. However, a secondary user has to leave this band and switch to another if the demand by primary users increases.

\footnotetext{
*Correspondence: jtadrous@rice.edu

1 Department of Electrical and Computer Engineering, Rice University, Houston, TX 77025, USA

Full list of author information is available at the end of the article
}

The notion of dynamic spectrum access has opened research in different problems regarding the new functionalities that a secondary user should perform, e.g., spectrum sensing, spectrum sharing, spectrum mobility, and spectrum management [2,3]. Moreover, information theoretic bounds on potential achievable rates by cognitive radio networks are being investigated. In most of those works, cooperation between primary and secondary transmitters is considered. In [4], an achievable rate region of primary versus secondary users' rates is introduced when a cognitive transmitter has full knowledge of the primary message in a two-transmitter two-receiver interference channel and the primary user cooperates with the secondary link through rate splitting introduced in [5]. In [6,7], the notion of conferencing is introduced for the interference channel where the cognitive link is assumed to know part or all of the message of the primary transmitter.

In this paper, we consider a primary multiple access channel (MAC) that consists of two transmitters and a

\section{Springer}

(c) 2014 Tadrous and Nafie; licensee Springer. This is an Open Access article distributed under the terms of the Creative Commons Attribution License (http://creativecommons.org/licenses/by/2.0), which permits unrestricted use, distribution, and reproduction in any medium, provided the original work is properly credited. 
common receiver shared by a secondary link comprising a single transmitter and a corresponding receiver. The secondary transmitter is assumed to employ rate splitting by dividing its signal into two parts: one part is decodable by the secondary receiver and treated as noise by the primary receiver, whereas the other part is decodable at both receivers. Such rate splitting scheme has also been suggested in [8] for a partially connected interference multiple access channel, with all users belonging to the same class of quality of service (QoS). The scheme has been shown to achieve the semi-deterministic capacity of the addressed setup to within a quantifiable gap. In [9], interference mitigation for a similar setup of interfering MAC has been considered. Authors have shown that signal scale alignment can be achieved through layered lattice codes, which potentially reduces interference by a factor of half for linear deterministic channels.

While we conduct our analysis for the discrete memoryless channel (DMC), we will give particular focus on the Gaussian setup, which is in essence similar to that discussed in $[10,11]$, with a primary multiple access network and a secondary transmitter-receiver pair. We investigate and characterize necessary and sufficient conditions under which interference cancellation (IC) at either primary or secondary users can strictly improve the performance of the achievable rates. Namely, we determine the case when the primary is able to cancel the interference of the secondary while not deteriorating the QoS for the secondary network. We also determine the case when the secondary can completely decode and cancel the interference of at least one primary transmitter while not hurting the primary achievable rates. In particular, we

- State the achievable rate region $\mathcal{R}^{o}$ in the DMC assuming that all of the primary signals are treated as noise at the secondary receiver

- State the achievable rate region $\mathcal{R}_{i}^{r}$, where the signal of primary transmitter $i$ is to be fully decodable at the secondary receiver besides being decodable at the primary receiver

- Show that there exists a case in which $\mathcal{R}_{i}^{r}$ contains $\mathcal{R}^{o}$

- Analyze the effect of rate splitting in a Gaussian setup where a necessary and sufficient condition is determined so that the union of the above regions is obtained without rate splitting

- Derive a necessary and sufficient condition so that the secondary receiver can decode the signal of a primary user without affecting the range of achievable primary sum rates, but only enhances the range of achievable secondary rates. We call this condition primary decodability condition for Gaussian (PDCG) channel

- Show, numerically, that the probability of having at least one primary user satisfying PDCG monotonically increases with the signal-to-noise ratio of the primary users

We conduct our analysis assuming a Gaussian communication channel as in [10], but for general channel gains, and adoption of rate splitting techniques. Some of the results in this paper have been presented in [11]. The introduced network model of a MAC primary network shared by secondary operations has been addressed in some resource allocation frameworks without rate splitting by secondary users [12-16]. Rate splitting by a secondary link, however, has been introduced in [17] where the secondary user is assumed to know the codebook of a primary transmitter and opportunistically splits its rate into two parts and decodes it in the following way. It decodes the first part treating both the primary signal and the second part as noise, decodes and cancels the primary signal, and then decodes the second part. This scheme is generalized in this paper as we consider the cases when the signal of one primary transmitter is decodable at the secondary receiver and when all the primary signals are treated as noise.

The rest of this paper is organized as follows. In Section 2, the DMC models are defined. In Section 3, the achievable rate regions are established for the defined DMC models. Then, obtained results are applied in a Gaussian channel setup in Section 4, and the paper is concluded in Section 5.

\section{Channel model}

In our formulation, we denote random variables by $X$, $Y, \cdots$ with realizations $x, y, \cdots$ from sets $\mathcal{X}, \mathcal{Y}, \cdots$, respectively. The communication channel is considered to be discrete and memoryless.

\subsection{Basic channel model}

We consider a basic channel $C_{B}$ defined by a tuple $\left(\mathcal{X}_{1}, \mathcal{X}_{2}, \mathcal{X}_{s}, \omega, \mathcal{Y}_{p}, \mathcal{Y}_{s}\right)$, where $\mathcal{X}_{1}, \mathcal{X}_{2}$ are two finite input alphabet sets of the primary transmitters and $\mathcal{X}_{s}$ is a finite input alphabet set of the secondary transmitter. Sets $\mathcal{Y}_{p}$ and $\mathcal{Y}_{s}$ are two finite output alphabet sets at the primary and secondary receivers, respectively, and $\omega$ is a collection of conditional channel probabilities $\omega\left(y_{p} y_{s} \mid x_{1} x_{2} x_{s}\right)$ of $\left(y_{p}, y_{s}\right) \in \mathcal{Y}_{p} \times \mathcal{Y}_{s}$ given $\left(x_{1}, x_{2}, x_{s}\right) \in \mathcal{X}_{1} \times \mathcal{X}_{2} \times \mathcal{X}_{s}$, with marginal conditional distributions:

$$
\omega_{a}\left(y_{a} \mid x_{1} x_{2} x_{s}\right)=\sum_{y_{a}^{*} \in \mathcal{Y}_{a}^{*}, a^{*} \neq a} \omega\left(y_{p} y_{s} \mid x_{1} x_{2} x_{s}\right), \quad a \in\{s, p\} .
$$

Since the channel is memoryless, the conditional probability $\omega^{n}\left(\mathbf{y}_{p} \mathbf{y}_{s} \mid \mathbf{x}_{1} \mathbf{x}_{2} \mathbf{x}_{s}\right)$ is given by

$$
\omega^{n}\left(\mathbf{y}_{p} \mathbf{y}_{s} \mid \mathbf{x}_{1} \mathbf{x}_{2} \mathbf{x}_{s}\right)=\prod_{t=1}^{n} \omega\left(y_{p}^{(t)} y_{s}^{(t)} \mid x_{1}^{(t)} x_{2}^{(t)} x_{s}^{(t)}\right)
$$


where

$$
\begin{aligned}
& \mathbf{x}_{a}=\left(x_{a}^{(1)}, \cdots, x_{a}^{(n)}\right) \in \mathcal{X}_{a}^{n}, a=1,2, s, \\
& \mathbf{y}_{a}=\left(y_{a}^{(1)}, \cdots, y_{a}^{(n)}\right) \in \mathcal{Y}_{a}^{n}, a=p, s .
\end{aligned}
$$

The same also holds for the marginal conditional distributions $\omega_{p}^{n}\left(\mathbf{y}_{p} \mid \mathbf{x}_{1} \mathbf{x}_{2} \mathbf{x}_{s}\right)$ and $\omega_{s}^{n}\left(\mathbf{y}_{s} \mid \mathbf{x}_{1} \mathbf{x}_{2} \mathbf{x}_{s}\right)$. Let $\mathcal{M}_{1}=$ $\left\{1, \cdots, M_{1}\right\}, \mathcal{M}_{2}=\left\{1, \cdots, M_{2}\right\}$ be message sets for primary transmitters 1 and 2 , respectively, and $\mathcal{M}_{s}=$ $\left\{1, \cdots, M_{s}\right\}$ be a message set for the secondary transmitter. A code $\left(n, M_{1}, M_{2}, M_{s}, \epsilon\right)$ is a collection of $M_{1}, M_{2}$, and $M_{s}$ codewords such that

1. Sender a, $a=1,2, s$, has an encoding function $\phi_{a}: i \rightarrow \mathbf{x}_{a i}, i \in \mathcal{M}_{a}$ and $\mathbf{x}_{a i} \in \mathcal{X}^{n}$

2. The primary receiver has $M_{1} M_{2}$ disjoint decoding sets $\mathcal{D}_{p i j} \subseteq \mathcal{Y}_{p}^{n}, i j \in \mathcal{M}_{1} \times \mathcal{M}_{2}$ and a decoding function $\psi_{p}: \mathbf{y}_{p} \rightarrow i j$ if $\mathbf{y}_{p} \in \mathcal{D}_{p i j}$, where $i j \in \mathcal{M}_{1} \times \mathcal{M}_{2}$

3. The secondary receiver has $M_{s}$ disjoint decoding sets $\mathcal{D}_{s k} \subseteq \mathcal{Y}_{s}^{n}, k \in \mathcal{M}_{s}$ and a decoding function $\psi_{s}: \mathbf{y}_{s} \rightarrow k$ if $\mathbf{y}_{s} \in \mathcal{D}_{s k}$, where $k \in \mathcal{M}_{s}$ (see Figure 1)

$$
\begin{gathered}
P e_{p}=\frac{1}{M_{1} M_{2} M_{s}} \sum_{i, j, k} \omega_{p}^{n}\left(\mathbf{y}_{p} \notin \mathcal{D}_{p i j} \mid \mathbf{x}_{1 i} \mathbf{x}_{2 j} \mathbf{x}_{s k}\right), \\
P e_{s}=\frac{1}{M_{1} M_{2} M_{s}} \sum_{i, j, k} \omega_{s}^{n}\left(\mathbf{y}_{s} \notin \mathcal{D}_{s k} \mid \mathbf{x}_{1 i} \mathbf{x}_{2 j} \mathbf{x}_{s k}\right)
\end{gathered}
$$

4. Probability of error for the primary network and the secondary link is less than $\epsilon$, that is, $P e_{p} \leq \epsilon$ and $P e_{s} \leq \epsilon$, respectively, where

A rate tuple $\left(R_{1}, R_{2}, R_{s}\right)$ of nonnegative real values is achievable if for any $\eta>0,0<\epsilon<1$ there exists a code such that

$$
\frac{1}{n} \log M_{a} \geq R_{a}-\eta, \quad a=1,2, s,
$$

with sufficiently large $n$.

\subsection{Rate splitting channel}

Rate splitting channel, $C_{R S}$, is a modified version of the basic channel $C_{B}$, where $C_{R S}$ is defined by a tuple

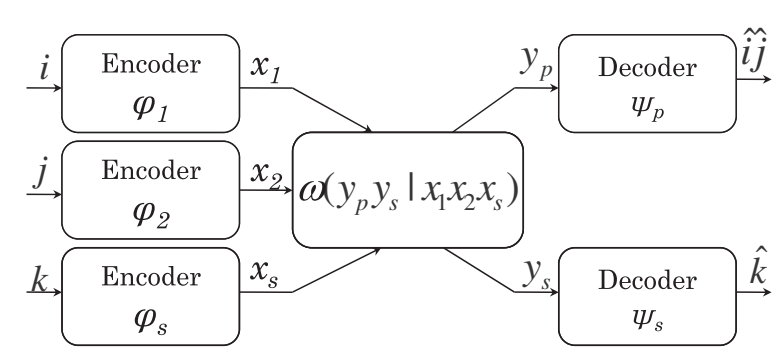

Figure 1 Basic channel model $C_{B}$.
$\left(\mathcal{X}_{1}, \mathcal{X}_{2}, \mathcal{X}_{s}, \omega, \mathcal{Y}_{p}, \mathcal{Y}_{s}\right)$ with its elements are as defined in $C_{B}$. Moreover, the input message sets for the primary transmitters are also $\mathcal{M}_{1}$ and $\mathcal{M}_{2}$ exactly as in $C_{B}$. However, the secondary user is assumed to have two finite message sets $\mathcal{L}_{s}=\left\{1, \cdots, L_{s}\right\}, \mathcal{N}_{s}=\left\{1, \cdots, N_{s}\right\}$. Hence, a code $\left(n, M_{1}, M_{2}, L_{s}, N_{s}, \epsilon\right)$ over the channel $C_{R S}$ is a collection of $M_{1}, M_{2}, L_{s} N_{s}$ codewords such that

1. Primary transmitter a, $a=1,2$, has an encoding function $\phi_{a}: i \rightarrow \mathbf{x}_{a i}, i \in \mathcal{M}_{a}, \mathbf{x}_{a i} \in \mathcal{X}_{a}^{n}$

2. The secondary transmitter has an encoding function $\phi_{s}: k l \rightarrow \mathbf{x}_{s k l}, k l \in \mathcal{L}_{s} \times \mathcal{N}_{s}, \mathbf{x}_{s k l} \in \mathcal{X}_{s}^{n}$

3. The primary receiver has $M_{1} M_{2} N_{s}$ disjoint decoding sets $\mathcal{D}_{p i j l} \subseteq \mathcal{Y}_{p}^{n}, i j l \in \mathcal{M}_{1} \times \mathcal{M}_{2} \times \mathcal{N}_{s}$ and a decoding function $\psi_{p}: \mathbf{y}_{p} \rightarrow i j l$ if $\mathbf{y}_{p} \in \mathcal{D}_{p i j l}$, where $i j l \in \mathcal{M}_{1} \times \mathcal{M}_{2} \times \mathcal{N}_{s}$

4. The secondary receiver has $L_{s} N_{s}$ disjoint decoding sets $\mathcal{D}_{s k l} \subseteq \mathcal{Y}_{s}^{n}, k l \in \mathcal{L}_{s} \times \mathcal{N}_{s}$ and a decoding function $\psi_{s}: \mathbf{y}_{p} \rightarrow k l$ if $\mathbf{y}_{p} \in \mathcal{D}_{s k l}$, where $k l \in \mathcal{L}_{s} \times \mathcal{N}_{s}$ (see Figure 2)

5. Probability of error for primary network and secondary link is less than $\epsilon$, that is, $P e_{p}^{o} \leq \epsilon$ and $P e_{s}^{o} \leq \epsilon$, respectively, where

$$
\begin{aligned}
& P e_{p}^{o}=\frac{1}{M_{1} M_{2} L_{s} N_{s}} \sum_{i, j, k, l} \omega_{p}^{n}\left(\mathbf{y}_{p} \notin \mathcal{D}_{p i j l} \mid \mathbf{x}_{1 i} \mathbf{x}_{2 j} \mathbf{x}_{s k l}\right), \\
& P e_{s}^{o}=\frac{1}{M_{1} M_{2} L_{s} N_{s}} \sum_{i, j, k, l} \omega_{s}^{n}\left(\mathrm{y}_{s} \notin \mathcal{D}_{s k l} \mid \mathbf{x}_{1 i} \mathbf{x}_{2 j} \mathbf{x}_{s k l}\right)
\end{aligned}
$$

A rate tuple $\left(R_{1}, R_{2}, S, T\right)$ of nonnegative real values is achievable over the channel $C_{R S}$ if there exists a code $\left(n, M_{1}, M_{2}, L_{s}, N_{s}, \epsilon\right)$ such that for any arbitrary $0<\epsilon<1$ and $\eta>0$

$$
\begin{aligned}
\frac{1}{n} \log M_{a} & \geq R_{a}-\eta, \quad a \in\{1,2\}, \\
\frac{1}{n} \log L_{s} & \geq S-\eta, \\
\frac{1}{n} \log N_{s} & \geq T-\eta,
\end{aligned}
$$

with sufficiently large $n$.

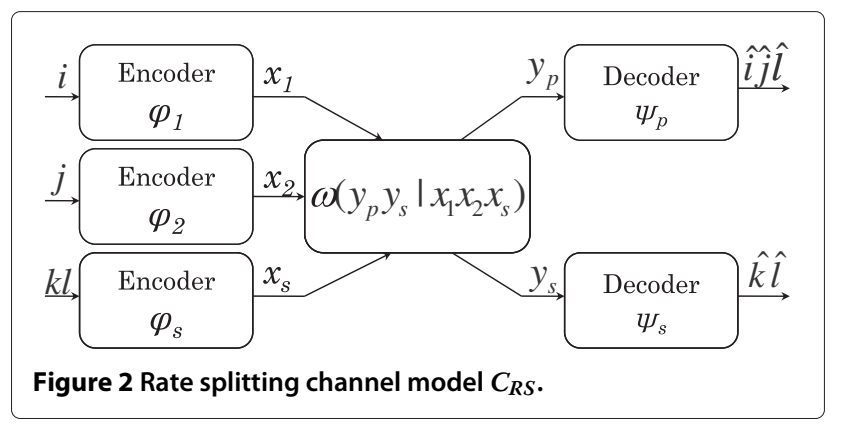


Lemma 1. If a rate tuple $\left(R_{1}, R_{2}, S, T\right)$ is achievable for $C_{R S}$, then a rate tuple $\left(R_{1}, R_{2}, R_{s}\right)$ where $R_{s}=S+T$ is achievable for $C_{B}$.

Proof. It is sufficient to show that if $\left(n, M_{1}, M_{2}, L_{s}, N_{s}, \epsilon\right)$ is a code for $C_{R S}$, then $\left(n, M_{1}, M_{2}, L_{s} N_{s}, \epsilon\right)$ is a code for $C_{B}$. To do so, let $\mathcal{D}_{p i j}=\cup_{l=1}^{N_{s}} \mathcal{D}_{\text {pijl }}$. Then,

$$
\omega_{p}^{n}\left(\mathbf{y}_{p} \notin \mathcal{D}_{p i j} \mid \mathbf{x}_{1 i} \mathbf{x}_{2 j} \mathbf{x}_{s k l}\right) \leq \omega_{p}^{n}\left(\mathbf{y}_{p} \notin \mathcal{D}_{p i j l} \mid \mathbf{x}_{1 i} \mathbf{x}_{2 j} \mathbf{x}_{s k l}\right) .
$$

So, if $\left(n, M_{1}, M_{2}, L_{s}, N_{s}, \epsilon\right)$ is a code for $C_{R S}$, then $P e_{p}^{o} \leq \epsilon$ and $P e_{s}^{o} \leq \epsilon$; hence, from (9), $P e_{p} \leq \epsilon$ and $P e_{s} \leq \epsilon$ when $k$ and $M_{s}$ of (1) are replaced with $k l$ and $L_{s} N_{s}$, respectively, meaning that $\left(n, M_{1}, M_{2}, L_{s} N_{s}, \epsilon\right)$ is a code for $C_{B}$.

\subsection{Rate splitting channel with decodable primary signal at the secondary receiver}

We introduce another channel, $C_{R S}^{p}$, in which the secondary user splits its set of messages into two sets, exactly as the case of $C_{R S}$. However, we assume that the signal of one primary transmitter is decodable at the secondary receiver. Without loss of generality, assume this is the first primary transmitter. Thus, $C_{R S}^{p}$ is defined by a tuple $\left(\mathcal{X}_{1}, \mathcal{X}_{2}, \mathcal{X}_{s}, \omega, \mathcal{Y}_{p}, \mathcal{Y}_{s}\right)$ with its elements defined as in $C_{B}$ and $C_{R S}$. A code for $C_{R S}^{p}$ is the same as in $C_{R S}$, except that conditions 4 and 5 are replaced by

4. Secondary receiver has $M_{1} L_{s} N_{s}$ disjoint decoding sets $\mathcal{D}_{s i k l} \subseteq \mathcal{Y}_{s}^{n}$ and a decoding function $\psi_{s}: \mathbf{y}_{s} \rightarrow i k l$ if $\mathbf{y}_{s} \in \mathcal{D}_{\text {sikl }}$, where $i k l \in \mathcal{M}_{1} \times \mathcal{L}_{s} \times \mathcal{N}_{s}$

5. Probability of error for the primary network and the secondary link is less than $\epsilon$, that is, $P e_{p}^{r} \leq \epsilon$ and $P e_{s}^{r} \leq \epsilon$, respectively, where

$$
\begin{aligned}
P e_{p}^{r} & =\frac{1}{M_{1} M_{2} L_{s} N_{s}} \sum_{i, j, k, l} \omega_{p}^{n}\left(\mathbf{y}_{p} \notin \mathcal{D}_{p i j l} \mid \mathbf{x}_{1 i} \mathbf{x}_{2 j} \mathbf{x}_{s k l}\right) \\
P e_{s}^{r} & =\frac{1}{M_{1} M_{2} L_{s} N_{s}} \sum_{i, j, k, l} \omega_{s}^{n}\left(\mathbf{y}_{s} \notin \mathcal{D}_{s i k l} \mid \mathbf{x}_{1 i} \mathbf{x}_{2 j} \mathbf{x}_{s k l}\right) .
\end{aligned}
$$

A rate tuple $\left(R_{1}, R_{2}, S, T\right)$ of nonnegative real values is achievable over the channel $C_{R S}^{p}$ if for any arbitrary $\eta>0$ and $0<\epsilon<1$, the inequalities (6) to (8) are satisfied for sufficiently large $n$.

Lemma 2. If a rate tuple $\left(R_{1}, R_{2}, S, T\right)$ is achievable for $C_{R S}^{p}$, then a rate tuple $\left(R_{1}, R_{2}, R_{s}\right)$ where $R_{s}=S+T$ is achievable for $C_{B}$.

Proof. The proof follows exactly as the proof of Lemma 1 noting that if $\mathcal{D}_{s k l}=\cup_{i=1}^{M_{1}} \mathcal{D}_{s i k l}$, then

$$
\omega_{s}^{n}\left(\mathbf{y}_{s} \notin \mathcal{D}_{s k l} \mid \mathbf{x}_{1 i} \mathbf{x}_{2 j} \mathbf{x}_{s k l}\right) \leq \omega_{s}^{n}\left(\mathbf{y}_{s} \notin \mathcal{D}_{s i k l} \mid \mathbf{x}_{1 i} \mathbf{x}_{2 j} \mathbf{x}_{s k l}\right) .
$$

At the end of this section, it is worth noting that $C_{B}$ furnishes a general structure for the communication setup of the system and does not explicitly pose any restrictions on the communication strategy used or limits the ability of certain receivers to decode the signals of noncorresponding transmitters. Yet, based on the primarysecondary nature of communication, we explicitly study special instances of $C_{B}$, in particular $C_{R S}$ and $C_{R S}^{p}$, in which the secondary user is capable of employing rate splitting, and potentially decode the signal of one primary user. Hence, it follows clearly that achievable rates for $C_{R S}$ and $C_{R S}^{p}$ are also achievable for $C_{B}$ as established in Lemmas 1 and 2.

\section{Achievable rate region}

In this section we investigate an achievable rate region for $C_{B}$. We first analyze two achievable rate regions, one for $C_{R S}$ and another for $C_{R S}^{p}$, and then state the overall achievable rate region. The random variables $U, W$, and $Q$ are defined over the finite sets $\mathcal{U}, \mathcal{W}$, and $\mathcal{Q}$, respectively, where $Q$ is a time-sharing parameter. Let the set $\mathcal{P}^{*}$ contain all $Z=Q U W X_{1} X_{2} X_{s} Y_{p} Y_{s}$ such that

- $X_{1}, X_{2}, U$, and $W$ are conditionally independent given $Q$

- $X_{s}=f(U W \mid Q)$

Since $X_{S}=f(\mathcal{W} \mid Q)$, then $\mathcal{U}$ and $\mathcal{W}$ can be considered as input sets to the channels $C_{R S}$ and $C_{R S}^{p}$.

\subsection{Achievable rate region for $C_{R S}$}

Theorem 1. For any $Z \in \mathcal{P}^{*}, \delta^{o}(Z)$ is the set of achievable rate tuples $\left(R_{1}, R_{2}, S, T\right)$ for $C_{R S}$ if the following inequalities are satisfied:

$$
\begin{array}{r}
R_{1} \leq I\left(Y_{p} ; X_{1} \mid W X_{2} Q\right), \\
R_{2} \leq I\left(Y_{p} ; X_{2} \mid W X_{1} Q\right), \\
T \leq I\left(Y_{p} ; W \mid X_{1} X_{2} Q\right), \\
R_{1}+R_{2} \leq I\left(Y_{p} ; X_{1} X_{2} \mid W Q\right), \\
T+R_{1} \leq I\left(Y_{p} ; W X_{1} \mid X_{2} Q\right), \\
T+R_{2} \leq I\left(Y_{p} ; W X_{2} \mid X_{1} Q\right), \\
T+R_{1}+R_{2} \leq I\left(Y_{p} ; W X_{1} X_{2} \mid Q\right) ; \\
S \leq I\left(Y_{s} ; U \mid W Q\right), \\
T \leq I\left(Y_{s} ; W \mid U Q\right), \\
S+T \leq I\left(Y_{s} ; U W \mid Q\right) .
\end{array}
$$

Proof. Please refer to Appendix 1.

Corollary 1. For $\delta^{o}=\cup_{Z \in \mathcal{P}^{*}} \delta^{o}(Z)$, any rate tuple of $\delta^{o}$ is achievable. 
We focus on the achievable rates by the primary network $R_{p}=R_{1}+R_{2}$ and the secondary link $R_{s}=S+T$. Let $\mathcal{R}^{o}(Z)$ be the set of all rate tuples $\left(R_{s}, R_{p}\right)$ having $\left(R_{1}, R_{2}, S, T\right)$ satisfy (13) to (22) for all $Z \in \mathcal{P}^{*}$, then the following theorem describes $\mathcal{R}^{o}(Z)$.

Theorem 2. For any $Z \in \mathcal{P}^{*}$, the achievable rate region $\mathcal{R}^{o}(Z)$ of the defined channel $C_{R S}$ consists of all rate pairs $\left(R_{s}, R_{p}\right)$ that satisfy

$$
R_{p} \leq \rho_{p}^{o}, \quad R_{s} \leq \rho_{s}^{o}, \quad R_{s}+R_{p} \leq \rho_{s p}^{o}
$$

where

$\rho_{p}^{o}=I\left(Y_{p} ; X_{1} X_{2} \mid W Q\right)$,

$\rho_{s}^{o}=I\left(Y_{s} ; U \mid W Q\right)+\sigma^{*}$,

$\rho_{s p}^{o}=\rho_{p}^{o}+I\left(Y_{s} ; U \mid W Q\right)+\min \left\{I\left(Y_{s} ; W \mid Q\right), I\left(Y_{p}, W \mid Q\right)\right\}$,

and

$$
\sigma^{*}=\min \left\{I\left(Y_{p} ; W \mid X_{1} X_{2} Q\right), I\left(Y_{s} ; W \mid Q\right)\right\}
$$

Proof. The proof can follow systematically using the Fourier-Motzkin elimination scheme, yet we use a different approach that determines the rate tuples $\left(R_{s}, R_{p}\right)$ of the corner points of $\mathcal{R}^{o}(Z)$, which will essentially be utilized in the proofs of other statements in the rest of this work. To that end, we refer to Figure 3.

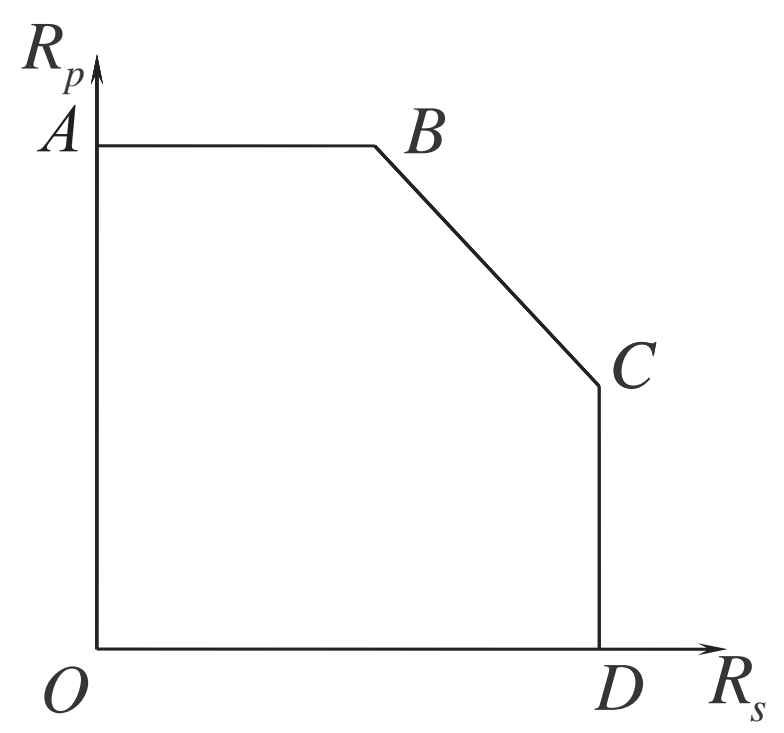

Figure 3 Achievable rate region $\mathcal{R}^{o}(Z)$ of the channel $C_{R S}$ for any $Z \in \mathcal{P}^{*}$.
- Point A:

$R_{s}^{A}=0$, i.e., $S^{A}=T^{A}=0$. Thus, the maximum rate at which the primary network can operate is determined from (16) as

$$
R_{p}^{A}=I\left(Y_{p} ; X_{1} X_{2} \mid W Q\right)=\rho_{p}^{o}
$$

- Point B:

At this point, we find the maximum possible rate at which the secondary user can transmit when the primary rate is $R_{p}^{B}=\rho_{p}^{o}$. In this case, the relations of (13) to (22) are reduced to

$$
\begin{aligned}
T & \leq I\left(Y_{p} ; W \mid Q\right), \\
\rho_{p}^{o}+T & \leq I\left(Y_{p} ; W X_{1} X_{2} \mid Q\right) ; \\
T & \leq I\left(Y_{s} ; W \mid U Q\right), \\
S & \leq I\left(Y_{s} ; U \mid W Q\right), \\
S+T & \leq I\left(Y_{s} ; U W \mid Q\right) .
\end{aligned}
$$

Since $T$ is irrelevant in (32), then $S$ can be set to

$$
S^{B}=I\left(Y_{s} ; U \mid W Q\right) .
$$

Hence, using chain rule in (30) and (33), the maximum value for $T$ would be

$$
T^{B}=\min \left\{I\left(Y_{p} ; W \mid Q\right), I\left(Y_{s} ; W \mid Q\right)\right\}
$$

and $R_{s}^{B}=S^{B}+T^{B}$.

- Point D:

$R_{1}^{D}=R_{2}^{D}=R_{p}^{D}=0$, then (13) to (22) are reduced to

$$
\begin{aligned}
T & \leq I\left(Y_{p} ; W \mid X_{1} X_{2} Q\right) ; \\
S & \leq I\left(Y_{s} ; U \mid W Q\right), \\
T & \leq I\left(Y_{s} ; W \mid U Q\right), \\
S+T & \leq I\left(Y_{s} ; U W \mid Q\right) .
\end{aligned}
$$

Since $T$ is irrelevant in (37), $S$ can be set to

$$
S^{D}=I\left(Y_{s} ; U \mid W Q\right) \text {. }
$$

Then,

$$
T^{D}=\sigma^{*}=\min \left\{I\left(Y_{s} ; W \mid Q\right), I\left(Y_{p} ; W \mid X_{1} X_{2} Q\right)\right\}
$$

and $R_{s}^{D}=S^{D}+T^{D}=\rho_{s}^{o}$.

- Point C:

At $R_{s}^{C}=\rho_{s}^{o}$, the maximum possible primary rate $R_{p}=$ $R_{1}+R_{2}$ has to satisfy

$$
\begin{aligned}
& R_{p} \leq I\left(Y_{p} ; X_{1} X_{2} \mid W Q\right), \\
& R_{p} \leq I\left(Y_{p} ; W X_{1} X_{2} \mid Q\right)-\sigma^{*} .
\end{aligned}
$$

Using chain rule, (43) can be rewritten as

$$
R_{p} \leq I\left(Y_{p} ; X_{1} X_{2} \mid W Q\right)+I\left(Y_{p} ; W \mid Q\right)-\sigma^{*}
$$


Thus, if $I\left(Y_{p} ; W \mid Q\right)-\sigma^{*}>0$, then (44) will be dominated by (42). Otherwise, (44) dominates (42). So, $R_{p}^{C}$ will be given by

$$
R_{p}^{C}=I\left(Y_{p} ; X_{1} X_{2} \mid W Q\right)-\left[\sigma^{*}-I\left(Y_{p} ; W \mid Q\right)\right]^{+}
$$

where $[x]^{+}=\max \{0, x\}$. The following is to show that both points $\left(R_{s}^{B}, R_{p}^{B}\right)$ and $\left(R_{s}^{C}, R_{p}^{C}\right)$ lie on the line $R_{s}+$ $R_{p}=\rho_{s p}^{o}$ :

For Point $\mathrm{B}$, using direct substitution with

$$
R_{s}^{B}=I\left(Y_{s} ; U \mid W Q\right)+\min \left\{I\left(Y_{p} ; W \mid Q\right), I\left(Y_{s} ; W \mid Q\right)\right\}
$$

and

$$
R_{p}^{B}=\rho_{p}^{o},
$$

it is clear that $R_{s}^{B}+R_{p}^{B}=\rho_{s p}^{o}$.

For Point $C$, we consider the following two possibilities:

- $\sigma^{*} \geq I\left(Y_{p} ; W \mid Q\right)$ :

Here $\min \left\{I\left(Y_{s} ; W \mid Q\right), I\left(Y_{p}, W \mid Q\right)\right\}=I\left(Y_{p} ; W \mid Q\right)$. Consequently,

$$
\rho_{s p}^{o}=I\left(Y_{s} ; U \mid W Q\right)+I\left(Y_{p} ; W X_{1} X_{2} \mid Q\right)
$$

and

$$
\begin{aligned}
& R_{s}^{C}+R_{p}^{C}=I\left(Y_{s} ; U \mid W Q\right)+I\left(Y_{p} ; W X_{1} X_{2} \mid Q\right) . \\
& \text { - } \sigma^{*}<I\left(Y_{p} ; W \mid Q\right):
\end{aligned}
$$

Since $I\left(Y_{p} ; W \mid X_{1} X_{2} Q\right) \geq I\left(Y_{p} ; W \mid Q\right)$, therefore $I\left(Y_{s}\right.$; $W \mid Q)<I\left(Y_{p} ; W \mid Q\right)$. Consequently,

$$
\rho_{s p}^{o}=I\left(Y_{s} ; U W \mid Q\right)+I\left(Y_{p} ; X_{1} X_{2} \mid W Q\right)
$$

and

$$
R_{s}^{C}+R_{p}^{C}=I\left(Y_{s} ; U W \mid Q\right)+I\left(Y_{p} ; X_{1} X_{2} \mid W Q\right) .
$$

Therefore, both rate tuples $\left(R_{s}^{B}, R_{p}^{B}\right)$ and $\left(R_{s}^{C}, R_{p}^{C}\right)$ lie on the line $R_{s}+R_{p}=\rho_{s p}^{o}$.

Note that, in the appendix of Han and Kobayashi [5], they argued that part of the achievable rate region by their introduced scheme was bounded by lines of slopes -0.5 and -2 . Although from (13) to (22) reducing $T$ by a value of $r$ may result in increase of $R_{p}$ by $2 r$, the proof that point $\left(R_{s}^{C}, R_{p}^{C}\right)$ lies on the line $R_{s}+R_{p}=\rho_{s p}^{o}$ means that a bound of slope -2 does not exist for $\mathcal{R}^{o}(Z)$.

Corollary 2. Any rate tuple $\left(R_{s}, R_{p}\right)$ of the region

$$
\mathcal{R}^{o}=\text { closure of } \bigcup_{Z \in \mathcal{P}^{*}} \mathcal{R}^{o}(Z)
$$

is achievable.

\subsection{Achievable rate region for $C_{R S}^{p}$}

Since in $C_{R S}^{p}$ the signal of one primary user has to be decodable at the secondary receiver, the model of $C_{R S}^{p}$ can be considered as the modified interference channel model, $C_{m}$, introduced in [5]. The signals of the two primary users can be treated as if they are produced from a single source, splitting its signal into two parts and encoding each part separately such that one part is decodable at both receivers while the other is decodable only at the primary receiver. For this channel, we define the set $\delta_{i}^{r}(Z)$ as the set of all achievable rate tuples $\left(R_{1}, R_{2}, S, T\right)$ when the signal of primary transmitter $i, i \in\{1,2\}$, is decodable by the secondary receiver. Without loss of generality, we assume that $i=1$. Hence, the achievable rate region for $C_{R S}^{p}$ takes the following form.

Theorem 3. For any $Z \in \mathcal{P}^{*}, \delta_{1}^{r}(Z)$ is the set of achievable rate tuples $\left(R_{1}, R_{2}, S, T\right)$ over the channel $C_{R S}^{p}$ if the following inequalities are satisfied:

$$
\begin{aligned}
R_{1} & \leq I\left(Y_{p} ; X_{1} \mid W X_{2} Q\right), \\
R_{2} & \leq I\left(Y_{p} ; X_{2} \mid W X_{1} Q\right), \\
T & \leq I\left(Y_{p} ; W \mid X_{1} X_{2} Q\right), \\
R_{1}+R_{2} & \leq I\left(Y_{p} ; X_{1} X_{2} \mid W Q\right), \\
R_{1}+T & \leq I\left(Y_{p} ; W X_{1} \mid X_{2} Q\right), \\
R_{2}+T & \leq I\left(Y_{p} ; W X_{2} \mid X_{1} Q\right), \\
R_{1}+R_{2}+T & \leq I\left(Y_{p} ; W X_{1} X_{2} Q\right) ; \\
S & \leq I\left(Y_{s} ; U \mid W X_{1} Q\right), \\
T & \leq I\left(Y_{s} ; W \mid U X_{1} Q\right), \\
R_{1} & \leq I\left(Y_{s} ; X_{1} \mid U W Q\right), \\
S+T & \leq I\left(Y_{s} ; U W \mid X_{1} Q\right), \\
R_{1}+S & \leq I\left(Y_{s} ; U X_{1} \mid W Q\right), \\
R_{1}+T & \leq I\left(Y_{s} ; W X_{1} \mid U Q\right), \\
R_{1}+S+T & \leq I\left(Y_{s} ; U W X_{1} \mid Q\right) .
\end{aligned}
$$

Proof. The proof follows exactly the proof of Theorem 3.1 in [5].

Corollary 3. For $\delta_{1}^{r}=\cup_{Z \in \mathcal{P}^{*}} \delta_{1}^{r}(Z)$, any rate tuple of $\delta_{1}^{r}$ is achievable.

For $C_{R S}^{p}$, the region $\mathcal{R}_{i}^{r}(Z)$ is the set of rate tuples $\left(R_{s}, R_{p}\right)$ where $R_{s}=S+T, R_{p}=R_{1}+R_{2}$, and $\left(R_{1}, R_{2}, S, T\right)$ is an element of $\delta_{i}^{r}(Z)$ for any $Z \in \mathcal{P}^{*}, i \in\{1,2\}$.

Theorem 4. For any $Z \in \mathcal{P}^{*}$, the achievable rate region $\mathcal{R}_{1}^{r}(Z)$ for the channel $C_{R S}^{p}$ consists of all rate pairs $\left(R_{s}, R_{p}\right)$ that satisfy

$$
\begin{aligned}
R_{s} \leq \rho_{s}^{r}, \quad R_{p} \leq \rho_{p}^{r}, & R_{s}+R_{p} \leq \rho_{s p}^{r}, \\
2 R_{s}+R_{p} \leq \rho_{2 p}^{r}, & R_{s}+2 R_{p} \leq \rho_{s 2}^{r}
\end{aligned}
$$


where

$$
\begin{aligned}
\rho_{s}^{r}= & I\left(Y_{s} ; U \mid W X_{1} Q\right)+\sigma_{s}^{*}, \\
\rho_{p}^{r}= & I\left(Y_{p} ; X_{2} \mid W X_{1} Q\right)+\sigma_{p}^{*}, \\
\rho_{s p}^{r}= & I\left(Y_{s} ; U \mid W X_{1} Q\right)+I\left(Y_{p} ; X_{2} \mid W X_{1} Q\right) \\
& +\min \left\{I\left(Y_{p} ; W X_{1} \mid Q\right), I\left(Y_{s} ; W X_{1} \mid Q\right),\right. \\
& I\left(Y_{p} ; W \mid X_{1} Q\right)+I\left(Y_{s} ; X_{1} \mid W Q\right), I\left(Y_{p} ; X_{1} \mid W Q\right) \\
& \left.+I\left(Y_{s} ; W \mid X_{1} Q\right)\right\}, \\
\rho_{2 p}^{r}= & 2 I\left(Y_{s} ; U \mid W X_{1} Q\right)+2 \sigma_{s}^{*}+I\left(Y_{p} ; X_{2} \mid W X_{1} Q\right) \\
& -\left[\sigma_{s}^{*}-I\left(Y_{p} ; W \mid X_{1} Q\right)\right]^{+}+\min \left\{I\left(Y_{s} ; X_{1} \mid W Q\right),\right. \\
& I\left(Y_{s} ; W X_{1} \mid Q\right)-\sigma_{s}^{*}, I\left(Y_{p} ; X_{1} \mid Q\right)+ \\
& {\left.\left[I\left(Y_{p} ; W \mid X_{1} Q\right)-\sigma_{s}^{*}\right]^{+}, I\left(Y_{p} ; X_{1} \mid W Q\right)\right\}, } \\
\rho_{s 2}^{r}= & 2 I\left(Y_{p} ; X_{2} \mid W X_{1} Q\right)+2 \sigma_{p}^{*}+I\left(Y_{s} ; U \mid W X_{1} Q\right)- \\
& {\left[\sigma_{p}^{*}-I\left(Y_{s} ; X_{1} \mid W Q\right)\right]^{+}+\min \left\{I\left(Y_{p} ; W \mid X_{1} Q\right),\right.} \\
& I\left(Y_{p} ; W X_{1} \mid Q\right)-\sigma_{p}^{*}, I\left(Y_{s} ; W \mid Q\right)+ \\
& {\left.\left[I\left(Y_{s} ; X_{1} \mid W Q\right)-\sigma_{p}^{*}\right]^{+}, I\left(Y_{s} ; W \mid X_{1} Q\right)\right\}, }
\end{aligned}
$$

and

$$
\begin{array}{r}
\sigma_{s}^{*}=\min \left\{I\left(Y_{s} ; W \mid X_{1} Q\right), I\left(Y_{p} ; W \mid X_{1} X_{2} Q\right)\right\}, \\
\sigma_{p}^{*}=\min \left\{I\left(Y_{p} ; X_{1} \mid W Q\right), I\left(Y_{s} ; X_{1} \mid U W Q\right)\right\}
\end{array}
$$

as shown in Figure 4.

Proof. From the similarity between $C_{R S}^{p}$ and the modified interference channel of Han and Kobayashi [5], the

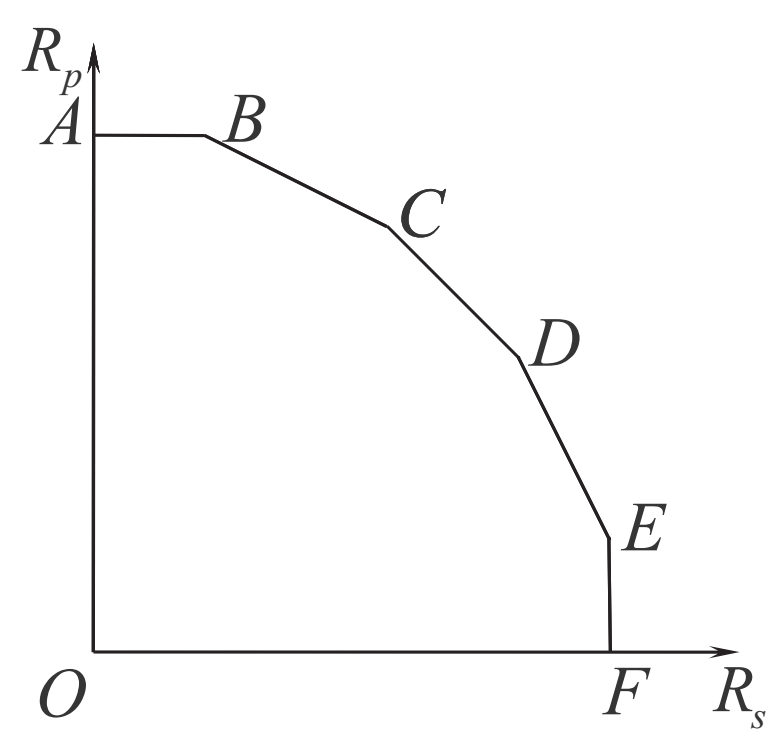

Figure 4 Achievable rate region $\mathcal{R}_{1}^{r}(Z)$ of the channel $C_{R S}^{p}$ for $Z \in \mathcal{P}^{*}$. derivation of the achievable rate region can be found in the appendix of [5]. The analysis goes as that done for $\mathcal{R}^{o}(Z)$ in $C_{R S}$. Hence, the corner points of the $\mathcal{R}_{1}^{r}(Z)$ are shown in Figure 4 and are given as follows.

- Point A:

$$
\begin{aligned}
& R_{s}^{A}=0, \\
& R_{p}^{A}=\rho_{p}^{r}=I\left(Y_{p} ; X_{2} \mid X_{1} W Q\right)+\sigma_{p}^{*} .
\end{aligned}
$$

- Point $B$ :

$$
\begin{aligned}
R_{s}^{B}= & I\left(Y_{s} ; U \mid W X_{1} Q\right)-\left[\sigma_{p}^{*}-I\left(Y_{s} ; X_{1} \mid W Q\right)\right]^{+} \\
& +\min \left\{I\left(Y_{p} ; W \mid X_{1} Q\right), I\left(Y_{p} ; W X_{1} \mid Q\right)-\sigma_{p}^{*},\right. \\
& I\left(Y_{s} ; W \mid Q\right)+\left[I\left(Y_{s} ; X_{1} \mid W Q\right)-\sigma_{p}^{*}\right]^{+}, \\
& \left.I\left(Y_{s} ; W \mid X_{1} Q\right)\right\} \\
R_{p}^{B}= & \rho_{p}^{r}=I\left(Y_{p} ; X_{2} \mid X_{1} W Q\right)+\sigma_{p}^{*} .
\end{aligned}
$$

- Point $C$ :

$$
\begin{aligned}
& R_{s}^{C}=2 \rho_{s p}^{r}-\rho_{s 2}^{r}, \\
& R_{p}^{C}=\rho_{s 2}^{r}-\rho_{s p}^{r} .
\end{aligned}
$$

- Point D:

$$
\begin{gathered}
R_{s}^{D}=\rho_{2 p}^{r}-\rho_{s p}^{r}, \\
R_{p}^{D}=2 \rho_{s p}^{r}-\rho_{s p}^{r} .
\end{gathered}
$$

- Point E:

$$
\begin{aligned}
R_{s}^{E}= & I\left(Y_{s} ; U \mid W X_{1} Q\right)+\sigma_{s}^{*}, \\
R_{p}^{E}= & I\left(Y_{p} ; X_{2} \mid W X_{1} Q\right)-\left[\sigma_{s}^{*}-I\left(Y_{p} ; W \mid X_{1} Q\right)\right]^{+} \\
& +\min \left\{I\left(Y_{s} ; X_{1} \mid W Q\right), I\left(Y_{s} ; W X_{1} \mid Q\right)-\sigma_{s}^{*},\right. \\
& I\left(Y_{p} ; X_{1} \mid Q\right)+\left[I\left(Y_{p} ; W \mid X_{1} Q\right)-\sigma_{s}^{*}\right]^{+}, \\
& \left.I\left(Y_{p} ; X_{1} \mid W Q\right)\right\} .
\end{aligned}
$$

- Point F:

$$
\begin{aligned}
R_{s}^{r F} & =\rho_{s}^{r}=I\left(Y_{s} ; U \mid W X_{1} Q\right)+\sigma_{s}^{*}, \\
R_{p}^{F} & =0 .
\end{aligned}
$$

Corollary 4. Any rate tuple $\left(R_{s}, R_{p}\right)$ of the regions

$$
\mathcal{R}_{i}^{r}=\text { closure } \bigcup_{Z \in \mathcal{P}^{*}} \mathcal{R}_{i}^{r}(Z), \quad i \in\{1,2\},
$$

is achievable.

Constraining the signal of one primary user to be decodable at the secondary receiver might result in a degradation in the achievable primary rate especially when the 
secondary rate is small. In general, $\mathcal{R}^{o}$ and $\mathcal{R}_{i}^{r}$ do not necessarily contain one another; however, there exists a case in which $\mathcal{R}^{o}(Z) \subseteq \mathcal{R}_{i}^{r}(Z)$. The following theorem characterizes that case.

Theorem 5. For a given $Z \in \mathcal{P}^{*}, \mathcal{R}^{o}(Z) \subseteq \mathcal{R}_{i}^{r}(Z)$ if and only if

$$
I\left(Y_{p} ; X_{i} \mid W Q\right) \leq I\left(Y_{s} ; X_{i} \mid U W Q\right) .
$$

Proof. Please refer to Appendix 2.

Corollary 5. If for all $Z \in \mathcal{P}^{*}$ condition (82) is satisfied, then $\mathcal{R}^{o} \subseteq \mathcal{R}_{i}^{r}$, where $\mathcal{R}_{i}^{r}=\cup_{Z \in \mathcal{P}^{*}} \mathcal{R}_{i}^{r}(Z)$.

Theorem 5 shows that when a primary user encodes its messages at a rate decodable at both receivers, the primary network may achieve the same rate range when none of the signals of its users is decodable at the secondary receiver. Moreover, at every primary rate, the secondary rate is enhanced (see Figure 5).

Consequently, if for any $Z \in \mathcal{P}^{*}$ condition (82) is satisfied, then allowing the secondary receiver to decode the signal of primary user $i$ at this $Z$ enhances the range of the secondary achievable rates without reducing the range of the achievable primary sum rates.

We call Corollary 5 the primary decodability condition (PDC).

\subsection{Achievable rate region for the channel $C_{B}$} From $C_{R S}$ and $C_{R S}^{p}$, we define

$$
\mathcal{R}_{i}(Z)=\mathcal{R}^{o}(Z) \cup \mathcal{R}_{i}^{r}(Z), \quad Z \in \mathcal{P}^{*}, i \in\{1,2\},
$$

and

$$
\mathcal{R}_{i}=\text { closure } \bigcup_{Z \in \mathcal{P}^{*}} \mathcal{R}_{i}(Z), \quad i \in\{1,2\} .
$$

Hence, an achievable rate region for the channel $C_{B}$

$$
\mathcal{R}=\mathcal{R}_{1} \cup \mathcal{R}_{2}
$$

or equivalently,

$$
\mathcal{R}=\mathcal{R}^{o} \cup \mathcal{R}_{1}^{r} \cup \mathcal{R}_{2}^{r} .
$$

At this point, it is worth reflecting the resulting achievable rate region $\mathcal{R}$ on the Han-Kobayashi region derived for the $2 \times 2$ interference channel, denoted $\mathcal{R}_{H K}$, especially with the adopted channel model $C_{B}$ which is well related to the interference channel $\mathbf{C}$ in [5]. In the light of the considered communication setup and adopted rate splitting communication scheme, we can note that the two primary transmitters of our setup $C_{B}$ can be viewed as a common transmitter in $\mathbf{C}$ splitting its signal into $X_{1}, X_{2}$.

However, since the transmitters are sending independent messages and having no control over the codebook of each other, their transmit strategies adopted in $\mathcal{R}$ can be considered as only three realizations of the possible rate splitting strategies for the common transmitter in C. Thus, we can note that $\mathcal{R} \subseteq \mathcal{R}_{H K}$. In particular, for

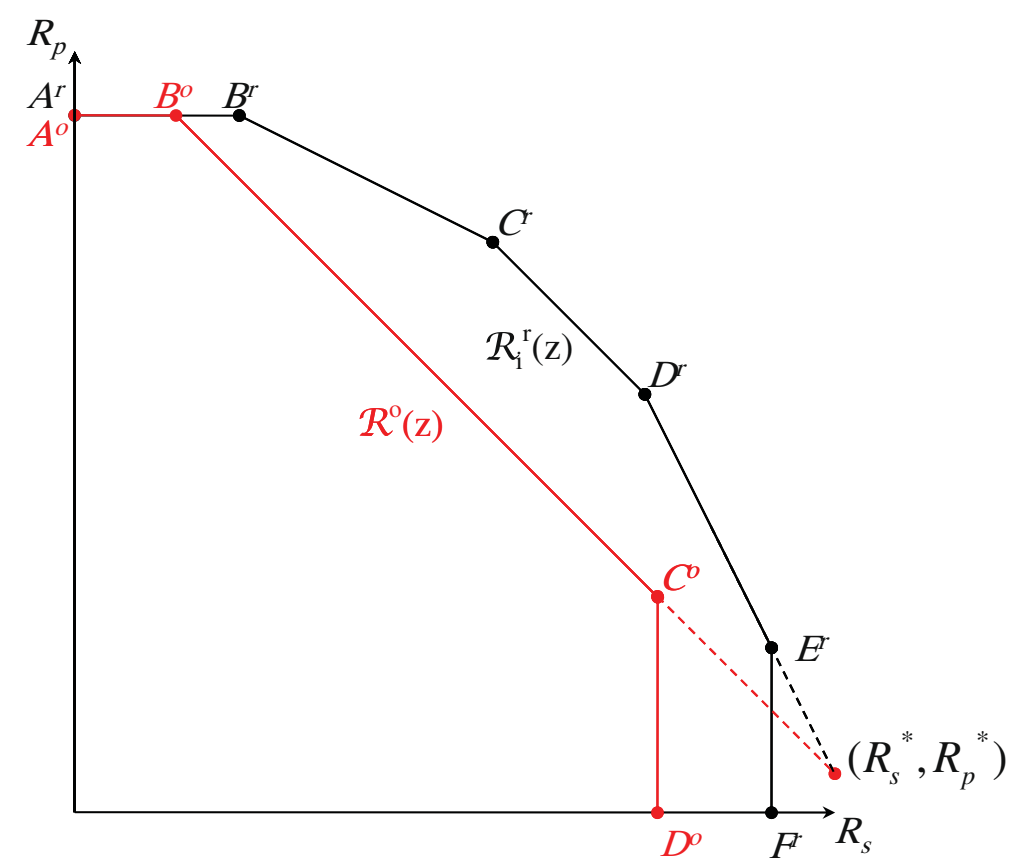

Figure 5 Regions $\mathcal{R}_{i}^{r}(Z)$ and $\mathcal{R}^{o}(Z)$ when $I\left(Y_{p} ; X_{i} \mid W Q\right) \leq I\left(Y_{s} ; X_{i} \mid U W Q\right)$. 
the two primary transmitters of $C_{B}$ behaving as a common transmitter in $\mathbf{C}$, rate region $\mathcal{R}$ spans only the rate splitting strategies of such common transmitter when the secondary receiver (1) cannot decode any primary signal, (2) can only decode the whole signal of user 1 , and (3) can only decode the whole signal of user 2 .

Note that, inequalities (15) and (49) used in $\delta^{o}(Z)$ and $\delta_{1}^{r}(Z)$, respectively, to limit the error in decoding the public part of the secondary signal at the primary receiver while the primary signals are decoded successfully. In fact, the primary receiver may not be interested in limiting the probability of such error event. Similarly, inequality (56) in $\delta_{1}^{r}(Z)$ may not be relevant as the secondary receiver is not interested in limiting the probability of error in decoding the primary signal when the two parts of its signal are decoded successfully. However, removing (15) from the definition of $\delta^{o}(Z)$ and (49) and (56) from the definition of $\delta_{1}^{r}(Z)$ does not enhance the achievable rate region $\mathcal{R}$.

To demonstrate this fact, we define $\delta^{\prime o}(Z)$ exactly as $\delta^{o}(Z)$ but without the constraint of $(15)$, and $\delta_{1}^{\prime r}(Z)$ exactly as $\delta_{1}^{r}(Z)$ but without the constraints (49) and (56). Let $\mathcal{R}^{\prime o}(Z)$ and $\mathcal{R}_{1}^{\prime r}(Z)$ be two sets of rate tuples $\left(R_{s}, R_{p}\right)$ such that $R_{s}=S+T$ and $R_{p}=R_{1}+R_{2}$, and the rate tuple $\left(R_{1}, R_{2}, S, T\right)$ is an element of $\delta^{\prime o}(Z)$ and $\delta_{1}^{\prime r}(Z)$, respectively. Also, we define

$$
\mathcal{R}_{1}^{\prime}(Z)=\mathcal{R}^{\prime o}(Z) \cup \mathcal{R}_{1}^{\prime r}(Z) .
$$

Theorem 6. If $\mathcal{R}_{1}^{\prime}=\bigcup_{Z \in \mathcal{P}^{*}} \mathcal{R}_{1}^{\prime}(Z)$, then $\mathcal{R}_{1}^{\prime}=\mathcal{R}_{1}$.

Proof. Please refer to Appendix 3.

\section{Corollary 6. For}

$$
\mathcal{R}^{\prime}=\text { closure of } \mathcal{R}_{1}^{\prime} \cup \mathcal{R}_{2}^{\prime} \text {, }
$$

then

$$
\mathcal{R}^{\prime}=\mathcal{R}
$$

\section{Gaussian channel}

In this section, we quantify the obtained achievable rate regions in a Gaussian channel model. A memoryless Gaussian channel of the introduced system is defined by a tuple $\left(\mathcal{X}_{1}, \mathcal{X}_{2}, \mathcal{X}_{s}, \omega, \mathcal{Y}_{p}, \mathcal{Y}_{s}\right)$ with $\mathcal{X}_{1}=\mathcal{X}_{2}=\mathcal{X}_{s}=$ $\mathcal{Y}_{p}=\mathcal{Y}_{s}=\Re$ (the field of real numbers), and a channel probability $\omega$ specified by

$$
\begin{aligned}
& y_{p}=\sqrt{g_{1}^{p}} x_{1}+\sqrt{g_{2}^{p}} x_{2}+\sqrt{g_{s}^{p}} x_{s}+n_{p}, \\
& y_{s}=\sqrt{g_{1}^{s}} x_{1}+\sqrt{g_{2}^{s}} x_{2}+\sqrt{g_{s}^{s}} x_{s}+n_{s}
\end{aligned}
$$

for $x_{1} \in \mathcal{X}_{1}, x_{2} \in \mathcal{X}_{2}, x_{s} \in \mathcal{X}_{s}, y_{p} \in \mathcal{Y}_{p}$, and $y_{s} \in \mathcal{Y}_{s}$, where $n_{p}$ and $n_{s}$ are independent Gaussian additive noise samples with zero mean and variance $N_{0}$, and $g_{1}^{p}, g_{2}^{p}, g_{s}^{p}, g_{1}^{s}$, $g_{2}^{s}$, and $g_{s}^{s}$ are the channel power gains. Power constraints are imposed on codewords $\mathbf{x}_{1}(i), \mathbf{x}_{2}(j), \mathbf{x}_{s}(k)\left(i \in \mathcal{M}_{1}, j \in\right.$ $\left.\mathcal{M}_{2}, k \in \mathcal{M}_{s}\right)$ :

$$
\begin{aligned}
& \frac{1}{n} \sum_{t=1}^{n}\left(x_{1}(i)^{(t)}\right)^{2}=P_{1}, \\
& \frac{1}{n} \sum_{t=1}^{n}\left(x_{2}(j)^{(t)}\right)^{2}=P_{2}, \\
& \frac{1}{n} \sum_{t=1}^{n}\left(x_{s}(k)^{(t)}\right)^{2}=P_{s} .
\end{aligned}
$$

Also, we define a subclass $\mathcal{G}\left(P_{1}, P_{2}, P_{s}\right)$ of $\mathcal{P}^{*}$ as follows: $Z=\phi U W X_{1} X_{2} X_{s} Y_{p} Y_{s} \in \mathcal{G}\left(P_{1}, P_{2}, P_{s}\right)$ if and only if $Z \in$ $\mathcal{P}^{*}, \sigma^{2}\left(X_{1}\right)=P_{1}, \sigma^{2}\left(X_{2}\right)=P_{2}$, and $\sigma^{2}\left(X_{s}\right)=P_{s}$, with $X_{1}$, $X_{2}, U$, and $W$ being zero mean Gaussian and $X_{s}=U+W$. Hence, we have the following rate regions achievable:

$$
\begin{aligned}
& \mathcal{R}_{g}^{o}=\text { closure of } \bigcup_{Z \in \mathcal{G}\left(P_{1}, P_{2}, P_{s}\right)} \mathcal{R}^{o}(Z), \\
& \mathcal{R}_{i g}^{r}=\text { closure of } \bigcup_{Z \in \mathcal{G}\left(P_{1}, P_{2}, P_{s}\right)} \mathcal{R}_{i}^{r}(Z), i \in\{1,2\}, \\
& \mathcal{R}_{i g}=\text { closure of } \bigcup_{Z \in \mathcal{G}\left(P_{1}, P_{2}, P_{s}\right)} \mathcal{R}_{i}(Z), i \in\{1,2\}, \\
& \mathcal{R}_{g}=\mathcal{R}_{g}^{o} \bigcup\left(\cup_{i \in\{1,2\}} \mathcal{R}_{i g}^{r}\right)=\mathcal{R}_{1 g} \bigcup \mathcal{R}_{2 g} .
\end{aligned}
$$

Assume the secondary user splits its power into $\lambda P_{s}$ and $\bar{\lambda} P_{s}$ such that $0 \leq \lambda \leq 1$ and $\lambda+\bar{\lambda}=1$. The part of secondary signal decodable at the primary and secondary receivers is encoded with power $\bar{\lambda} P_{s}$ where the other part is encoded with power $\lambda P_{s}$. Let $\tau(x)=0.5 \log _{2}(1+x)$, and the relevant quantities in Theorems 2 and 4 will be given by

$$
\begin{aligned}
& I\left(Y_{p} ; X_{1} X_{2} \mid W\right)=\tau\left(\frac{g_{1}^{p} P_{1}+g_{2}^{p} P_{2}}{g_{s}^{p} \lambda P_{s}+N_{0}}\right), \\
& I\left(Y_{p} ; X_{1} X_{2}\right)=\tau\left(\frac{g_{1}^{p} P_{1}+g_{2}^{p} P_{2}}{g_{s}^{p} P_{s}+N_{0}}\right), \\
& I\left(Y_{p} ; X_{2} \mid W X_{1}\right)=\tau\left(\frac{g_{2}^{p} P_{2}}{g_{s}^{p} \lambda P_{s}+N_{0}}\right), \\
& I\left(Y_{p} ; X_{1} \mid W\right)=\tau\left(\frac{g_{1}^{p} P_{1}}{g_{s}^{p} \lambda P_{s}+g_{2}^{p} P_{2}+N_{0}}\right), \\
& I\left(Y_{p} ; W \mid X_{1} X_{2}\right)=\tau\left(\frac{g_{s}^{p} \bar{\lambda} P_{s}}{g_{s}^{p} \lambda P_{s}+N_{0}}\right), \\
& I\left(Y_{p} ; W \mid X_{1}\right)=\tau\left(\frac{g_{s}^{p} \bar{\lambda} P_{s}}{g_{s}^{p} \lambda P_{s}+g_{2}^{p} P_{2}+N_{0}}\right),
\end{aligned}
$$




$$
\begin{aligned}
& I\left(Y_{p} ; W X_{1}\right)=\tau\left(\frac{g_{1}^{p} P_{1}+g_{s}^{p} \bar{\lambda} P_{s}}{g_{s}^{p} \lambda P_{s}+g_{2}^{p} P_{2}+N_{0}}\right), \\
& I\left(Y_{p} ; W\right)=\tau\left(\frac{g_{s}^{p} \bar{\lambda} P_{s}}{g_{s}^{p} \lambda P_{s}+g_{1}^{p} P_{1}+g_{2}^{p} P_{2}+N_{0}}\right) \\
& I\left(Y_{p} ; X_{1}\right)=\tau\left(\frac{g_{1}^{p} P_{1}}{g_{s}^{p} P_{s}+g_{2}^{p} P_{2}+N_{0}}\right) ; \\
& I\left(Y_{s} ; U \mid W X_{1}\right)=\tau\left(\frac{g_{s}^{s} \lambda P_{s}}{g_{2}^{s} P_{2}+N_{0}}\right), \\
& I\left(Y_{s} ; U \mid W\right)=\tau\left(\frac{g_{s}^{s} \lambda P_{s}}{g_{1}^{s} P_{1}+g_{2}^{s} P_{2}+N_{0}}\right), \\
& I\left(Y_{s} ; W \mid X_{1}\right)=\tau\left(\frac{g_{s}^{s} \bar{\lambda} P_{s}}{g_{s}^{s} \lambda P_{s}+g_{2}^{s} P_{2}+N_{0}}\right), \\
& I\left(Y_{s} ; W X_{1}\right)=\tau\left(\frac{g_{s}^{s} \bar{\lambda} P_{s}+g_{1}^{s} P_{1}}{g_{s}^{s} \lambda P_{s}+g_{2}^{s} P_{2}+N_{0}}\right), \\
& I\left(Y_{s} ; W\right)=\tau\left(\frac{g_{s}^{s} \bar{\lambda} P_{s}}{g_{s}^{s} \lambda P_{s}+g_{1}^{s} P_{1}+g_{2}^{s} P_{2}+N_{0}}\right), \\
& I\left(Y_{s} ; X_{1} \mid W\right)=\tau\left(\frac{g_{1}^{s} P_{1}}{g_{s}^{s} \lambda P_{s}+g_{2}^{s} P_{2}+N_{0}}\right), \\
& I\left(Y_{s} ; X_{1} \mid U W\right)=\tau\left(\frac{g_{1}^{s} P_{1}}{g_{2}^{s} P_{2}+N_{0}}\right) .
\end{aligned}
$$

\subsection{Performance of rate splitting}

We study the effect of rate splitting by the secondary link on the achievable rate regions $\mathcal{R}_{g}^{o}$ and $\mathcal{R}_{i g}^{r}, i \in\{1,2\}$ and hence $\mathcal{R}_{i g}$. For each region, there exists a case for which no rate splitting determines the overall region, i.e., each achievable rate region is obtained at $\lambda=0$ or $\lambda=1$. We say that rate splitting does not affect an achievable rate region $\mathcal{A}$ if $\mathcal{A}(Z)$ coincides on $\mathcal{A}$ at $\lambda=0$ or $\lambda=1$, $Z \in \mathcal{G}\left(P_{1}, P_{2}, P_{s}\right)$, where $\mathcal{A}=\bigcup_{Z \in \mathcal{G}\left(P_{1}, P_{2}, P_{s}\right)} \mathcal{A}(Z)$, meaning that either decoding the whole secondary signal at the primary receiver or not decoding it at all determines $\mathcal{A}$.

\subsubsection{For $\mathcal{R}_{g}^{o}$}

The region $\mathcal{R}_{g}^{o}$ is obtained when the secondary receiver is assumed to treat the primary interference as noise. The following theorem determines the effect of rate splitting on $\mathcal{R}_{g}^{o}$.

Theorem 7. For $Z \in \mathcal{G}\left(P_{1}, P_{2}, P_{s}\right)$, an achievable rate region $\mathcal{R}^{o}(Z)$ can only coincide on $\mathcal{R}_{g}^{o}$ at $\lambda=0$, if and only if

$$
I\left(Y_{s} ; W\right) \leq I\left(Y_{p} ; W \mid X_{1} X_{2}\right)
$$

or equivalently,

$$
g_{s}^{s} N_{0} \leq g_{s}^{p}\left(g_{1}^{s} P_{1}+g_{2}^{s} P_{2}+N_{0}\right) .
$$

Proof. Please refer to Appendix 4.
Theorem 7 shows that rate splitting does not affect the achievable rate region $\mathcal{R}_{g}^{o}$ when inequality (97) is satisfied. Hence, a primary receiver decoding all the secondary signal is preferable at this case. Figure 6 depicts this case for different values of $\lambda$. It is clear that $\mathcal{R}^{o}(Z)$ at smaller $\lambda$ contains $\mathcal{R}^{o}(Z)$ at larger $\lambda$. This figure was obtained at $g_{1}^{p}=2.5664, g_{2}^{p}=3.7653, g_{1}^{s}=0.1812, g_{2}^{s}=0.1784$, $g_{s}^{p}=2.3620$, and $g_{s}^{s}=8.6065$ and at the following power setup. The noise variance $N_{0}=1$ unit power and $\frac{P_{1}}{N_{0}}=$ $\frac{P_{2}}{N_{0}}=S N R_{p}=10 \mathrm{~dB}$ and $\frac{P_{s}}{N_{0}}=S N R_{s}=10 \mathrm{~dB}$. Note that in this case, the maximum secondary throughput does not depend on $\lambda$, so the best performance from the primary rate point of view is to decode all the secondary signal by setting $\lambda=0$.

Moreover, when inequality (97) is not satisfied, rate splitting affects $\mathcal{R}_{g}^{o}$ as for any two different values of $\lambda$ the corresponding $\mathcal{R}^{o}(Z) \mathrm{s}$ do not contain one another. Hence, $\mathcal{R}_{g}^{o}$ is obtained by varying $\lambda$ from 0 to 1 . Figure 7 represents the case when (97) is not satisfied for the following parameters. $g_{1}^{p}=1.5066, g_{2}^{p}=0.8290, g_{1}^{s}=0.1902$, $g_{2}^{s}=0.0122, g_{s}=1.1953$, and $g_{s}^{s}=10.3229$ with the same power setup of Figure 6 .

Also, it is shown in [11] that when (97) is not satisfied, the sum throughput of the whole network, i.e., $R_{s}+R_{p}$, increases with $\lambda$. That is, as $\lambda$ increases, the primary sum rate decreases but the secondary rate gains an increase larger than the decrease in rate encountered by the primary network. Figure 8 depicts $R_{s}+R_{p}$ for the same simulation parameters of Figure 7. It is clear that the increase in the total sum rate, $R_{s}+R_{p}$, is accompanied by a decrease in the sum primary rate $R_{p}$. Hence, the sum primary rate has to be protected above a minimum limit.

\subsubsection{For $\mathcal{R}_{i g^{\prime}}^{r} i \in\{1,2\}$}

The region $\mathcal{R}_{i g}^{r}$ is obtained when the secondary receiver can decode the signal of primary user $i$. The rate splitting effect on this region is determined in the following theorem.

Theorem 8. For $Z \in \mathcal{G}\left(P_{1}, P_{2}, P_{s}\right)$ and $i \in\{1,2\}$, an achievable rate region $\mathcal{R}_{i}^{r}(Z)$ can only coincide on $\mathcal{R}_{i g}^{r}$ at $\lambda=0$ if and only if

$$
I\left(Y_{s} ; W \mid X_{i}\right) \leq I\left(Y_{p} ; W \mid X_{1} X_{2}\right)
$$

or equivalently,

$$
g_{s}^{s} N_{0} \leq g_{s}^{p}\left(g_{j}^{s} P_{j}+N_{0}\right), \quad j \in\{1,2\}, j \neq i .
$$

Proof. Please refer to Appendix 5.

Hence, if inequality (99) is satisfied, $\mathcal{R}_{i g}^{r}$ is obtained without rate splitting, specifically, when $\lambda=0$.

Figures 9 and 10 show the performance of rate splitting under same power setup used with Figures 6 and 7 where 


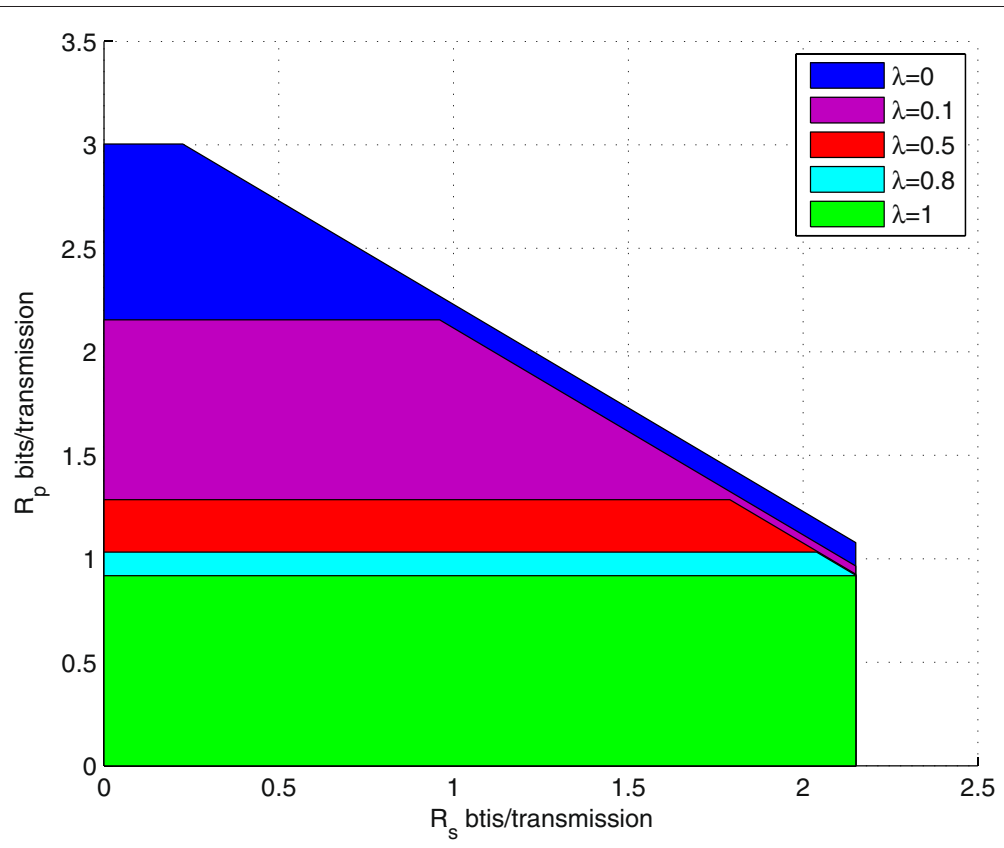

Figure 6 Overall achievable rate region $\mathcal{R}_{g}^{o}$ is obtained when the whole secondary signal is decodable by the primary receiver. $\mathcal{R}_{g}^{\circ}$ is

shown in blue.

it is assumed that the secondary receiver can decode the signal of primary user 1 . In Figure 9, the achievable rate region $\mathcal{R}_{1 g}^{r}$ coincides on $\mathcal{R}_{1}^{r}(Z)$ when inequality (99) is satisfied. The parameters for this scenario are $g_{1}^{p}=5.5303$, $g_{2}^{p}=4.2865, g_{1}^{s}=0.6542, g_{2}^{s}=0.8121, g_{s}^{p}=3.9334$, and $g_{s}^{s}=8.1575$.
In Figure 10, the opposite scenario is considered where inequality (99) is not satisfied. It is obvious that the overall rate region $\mathcal{R}_{1 g}^{r}$ is obtained by varying $\lambda$ from 0 to 1 as a consequence of the fact that rate regions corresponding to different values of $\lambda$ do not include one another if inequality (99) is not satisfied. The channel gains for Figure 10

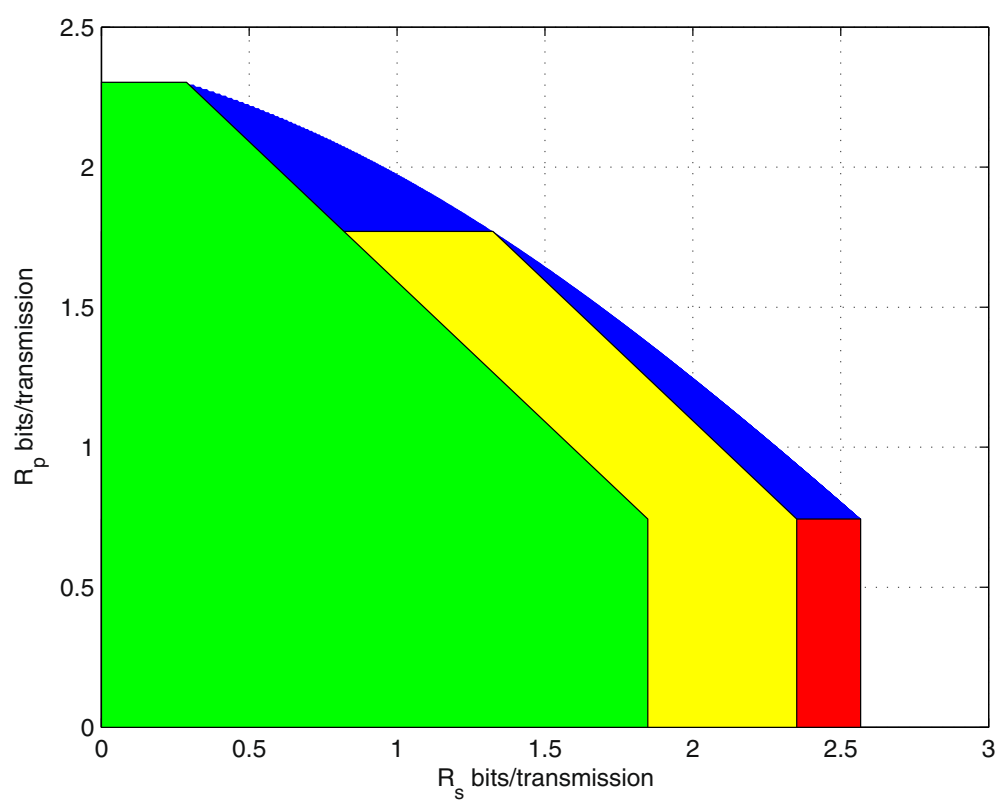

Figure 7 Rate splitting affects the achievable rate region. $\mathcal{R}_{g}^{\circ}$ is shown in blue, and $\boldsymbol{\mathcal { R }}^{\circ}(Z)$ is shown in green for $\lambda=0$, yellow for $\lambda=0.1$, and red for $\lambda=1$. 


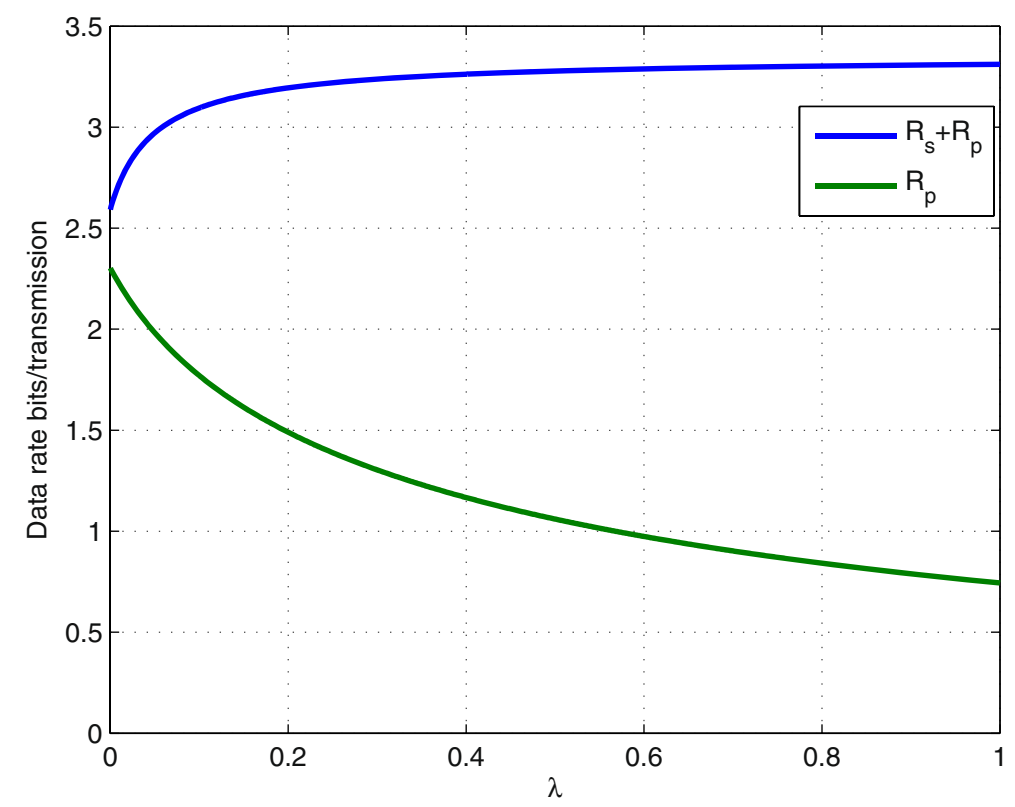

Figure 8 Increase in the sum rate of the whole network when inequality (97) is not satisfied.

$\underset{p}{\operatorname{are}} g_{1}^{p}=9.566, g_{2}^{p}=14.5045, g_{1}^{s}=0.0808, g_{2}^{s}=0.2894$, $g_{s}^{p}=0.7032$, and $g_{s}^{s}=16.6226$.

Consequently, the achievable rate region $\mathcal{R}_{\text {ig }}$ coincides on $\mathcal{R}_{i g}(Z)$ at $\lambda=0$ if and only if (99) is satisfied.

\subsection{Decoding one primary signal}

In Section 3.2, we have discussed the achievable rate region in the DMC case assuming that the signal of one primary transmitter has to be reliably decoded by the secondary receiver. Although this may impose a constraint on the range of achievable sum rates by the primary network, we showed in Theorem 5 and Corollary 5 that there exists a condition for which this constraint only enhances the achievable rates for the secondary link without degrading the range of achievable rates by the primary network. This condition is called PDC. When applying this condition to

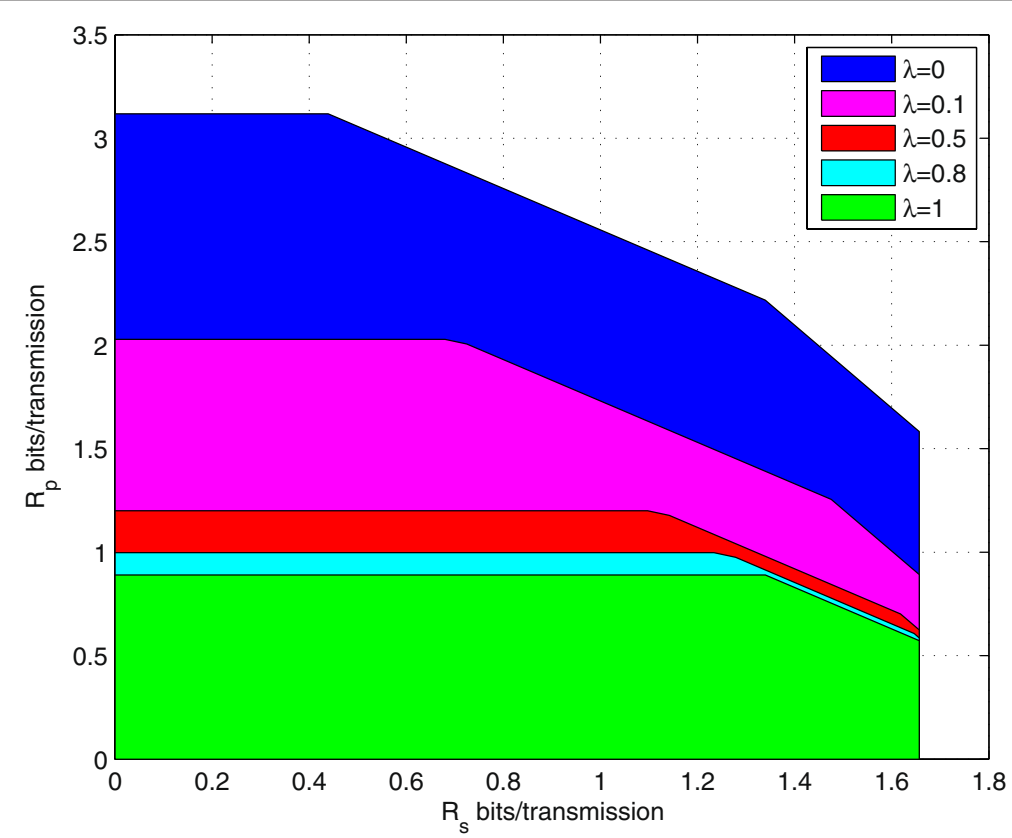

Figure 9 Overall achievable rate region $\mathcal{R}_{1 g}^{r}$ is obtained when the whole secondary signal is decodable by the primary receiver. $\mathcal{R}_{1 g}^{r}$ is shown in blue. 


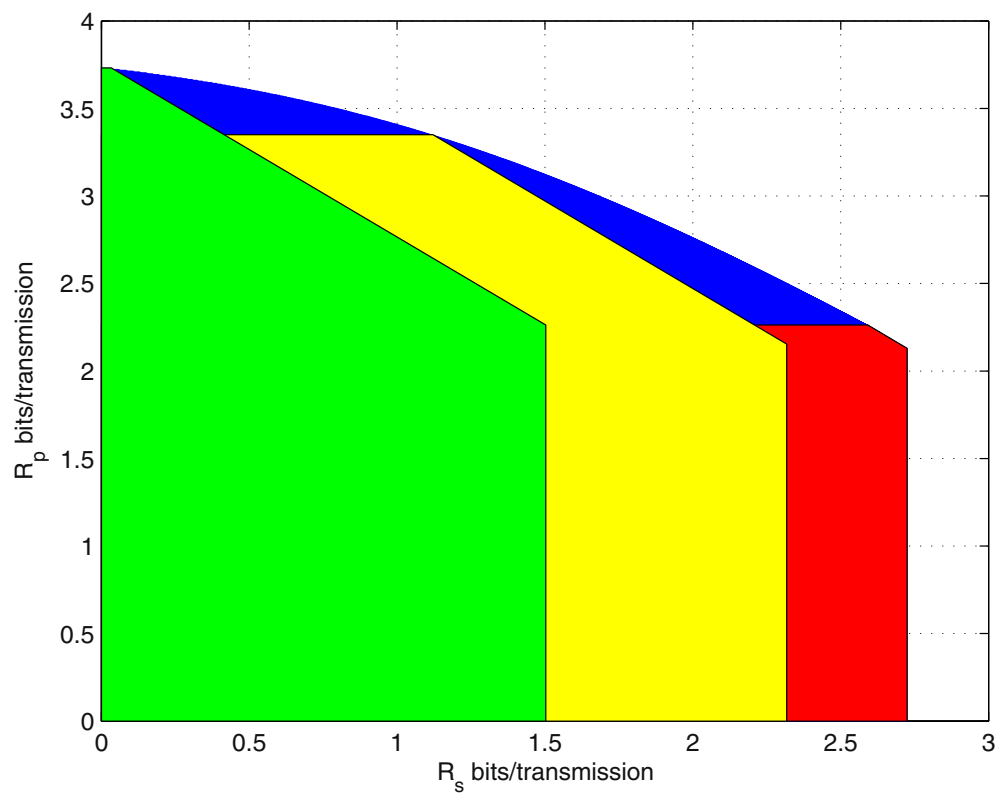

Figure 10 Rate splitting affects the achievable rate region. $\mathcal{R}_{1 g}^{r}$ is shown in blue, and $\boldsymbol{\mathcal { R }}_{1}^{r}(Z)$ is shown in green for $\lambda=0$, yellow for $\lambda=0.1$, and red for $\lambda=1$.

the given Gaussian channel, the PDC would be as follows: If for all $Z \in \mathcal{G}\left(P_{1}, P_{2}, P_{s}\right) I\left(Y_{p} ; X_{i} \mid W\right) \leq I\left(Y_{s} ; X_{i} \mid U W\right)$, then $\mathcal{R}_{g}^{o} \subseteq \mathcal{R}_{i g}^{r}$. Equivalently, the following inequality must hold:

$\tau\left(\frac{g_{i}^{p} P_{i}}{g_{s}^{p} \lambda P_{s}+g_{j}^{p} P_{j}+N_{0}}\right) \leq \tau\left(\frac{g_{i}^{s} P_{i}}{g_{j}^{s} P_{j}+N_{0}}\right)$,

$\forall \lambda: 0 \leq \lambda \leq 1, j \neq i, i, j \in\{1,2\}$.

But since $I\left(Y_{s} ; X_{i} \mid U W\right)$ does not depend on $\lambda$, then a necessary and sufficient condition to have (100) satisfied is

$$
\frac{g_{i}^{p}}{g_{j}^{p} P_{j}+N_{0}} \leq \frac{g_{i}^{s}}{g_{j}^{s} P_{j}+N_{0}}, \quad j \neq i, \quad i, j \in\{1,2\} .
$$

We call inequality (101) primary decodability condition for Gaussian channel (PDCG).

Figure 11 shows a scenario for which three rate regions are obtained: $\mathcal{R}_{g}^{o}, \mathcal{R}_{1 g}^{r}$, and $\mathcal{R}_{2 g}^{r}$. It is clear that $\mathcal{R}_{g}^{o} \subseteq \mathcal{R}_{1 g}^{r}$ meaning that primary user 1 satisfies the PDCG described in (101), whereas primary user 2 does not. By decoding the signal of primary user 1 at the secondary receiver, the range of achievable primary rates in $\mathcal{R}_{g}^{o}$ remains the same for $\mathcal{R}_{1 g}^{r}$ while the secondary link can achieve higher rate at a given primary rate in $\mathcal{R}_{1 g}^{r}$ than in $\mathcal{R}_{g}^{o}$. The power setup used to produce this figure is the same as that of Figure 6, and the channel gains are $g_{1}^{p}=0.3413, g_{2}^{p}=10.2047, g_{1}^{s}=$ $0.2821, g_{2}^{s}=0.3782, g_{s}^{p}=0.2495$, and $g_{s}^{s}=6.3337$.

Note that a primary user that satisfies PDCG does not always exist, so we evaluate the probability of PDCG as the probability of finding at least one primary user satisfying (101). We assume $N_{0}=1$ unit power and $g_{1}^{s}$ and $g_{2}^{s}$ are i.i.d. exponentially distributed with mean $\mu_{s}$, whereas $g_{1}^{p}$ and $g_{2}^{p}$ are i.i.d. exponentially distributed with mean $\mu_{p}$, where $g_{1}^{s}, g_{2}^{s}, g_{1}^{p}$, and $g_{2}^{p}$ are mutually independent. A closed form formula for the probability of PDCG is difficult to obtain, so we evaluate it numerically by generating $10^{7}$ different values for each channel gain element and calculating the average number of times at which neither primary user satisfies $(101)$ at a given $P_{1}$ and $P_{2}$; then by subtracting it from 1 , we get a numerical estimate for the probability of PDCG. A simulation has been done in which we assume that $\frac{P_{1}}{N_{0}}=\frac{P_{2}}{N_{0}}=S N R_{p}$. We vary $S N R_{p}$ and evaluate the corresponding probability of PDCG. This simulation is done for the following pairs of $\left(\mu_{p}, \mu_{s}\right):(1,1),(1,5),(5,1)$, and $(5,5)$. The result is shown in Figure 12, where it is obvious that the probability of PDCG increases with $S N R_{p}$ and that the increase in $\mu_{s}$ yields more increase in probability of PDCG. The monotonic increase of such probability with $S N R_{p}$ can be seen by explicitly expressing the probability of event (101) as $P\left(\frac{g_{j}^{s} S N R_{p}+1}{g_{j}^{p} S N R_{p}+1} \leq \frac{g_{i}^{s}}{g_{i}^{p}}\right.$, for some $\left.i, j \in\{1,2\}\right)$, which is essentially monotonically increasing in $S N R_{p}$ and approaches 1 as $S N R_{p}$ goes to $\infty$. While it is hard to mathematically show the dependence of the probability of PDCG on $\mu_{s}$ and $\mu_{p}$, we can justify the increase of such probability with $\mu_{s}$ relative to $\mu_{p}$ because it statistically implies higher quality of the channel to the secondary receiver than that to the primary, hence more chances of (101). 


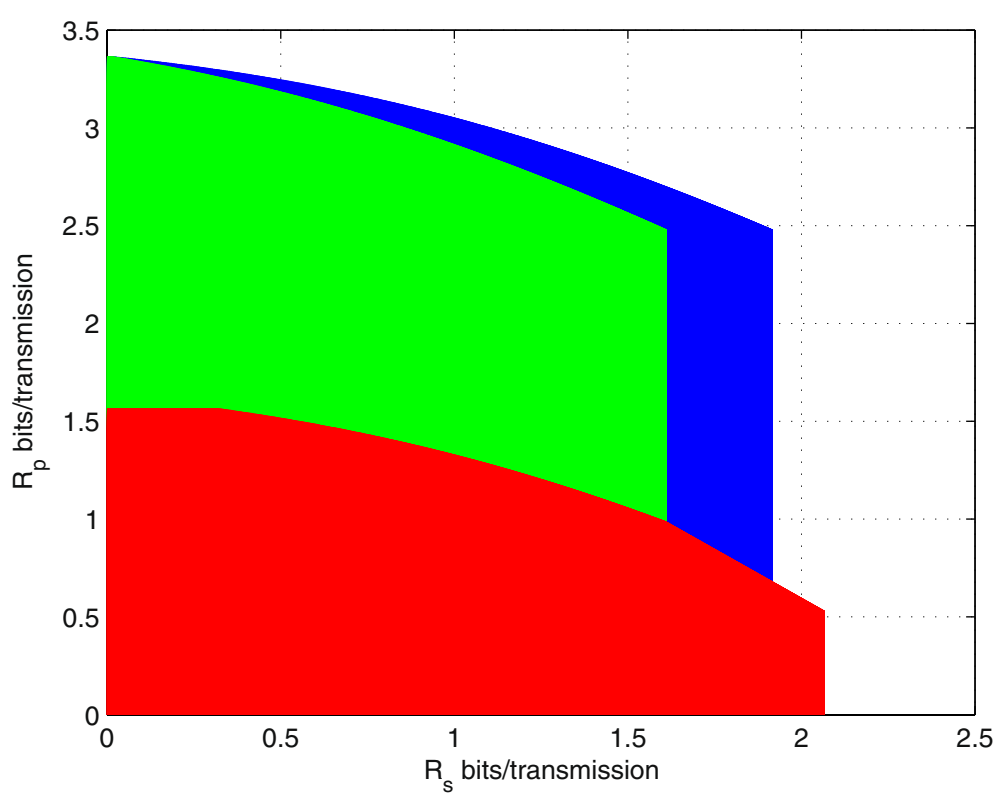

Figure 11 Achievable rate regions for the Gaussian channel. $\boldsymbol{\mathcal { R }}_{g}^{o}$ is shown in green, $\boldsymbol{\mathcal { R }}_{1 g}^{r}$ in blue, and $\boldsymbol{\mathcal { R }}_{2 g}^{r}$ in red.

\section{Conclusions}

In this work, we have analyzed an achievable rate region for a primary multiple access network coexisting with a secondary link that comprises one transmitter and a corresponding receiver. The achievable rate regions depict the sum primary rate versus the secondary rate. We have considered DMC where the secondary link employs rate splitting and investigated two types of achievable rate regions: the first is when the secondary receiver treats the primary signal as noise, whereas the second is when the secondary is able to decode the signal of only one primary transmitter. An overall achievable rate region is the union of those two regions. Moreover, we have shown that there exists a case for which allowing the secondary receiver to decode a primary signal results in an achievable rate region that includes the achievable rate region obtained when the secondary receiver does not decode the primary signal. Subsequently, we have investigated

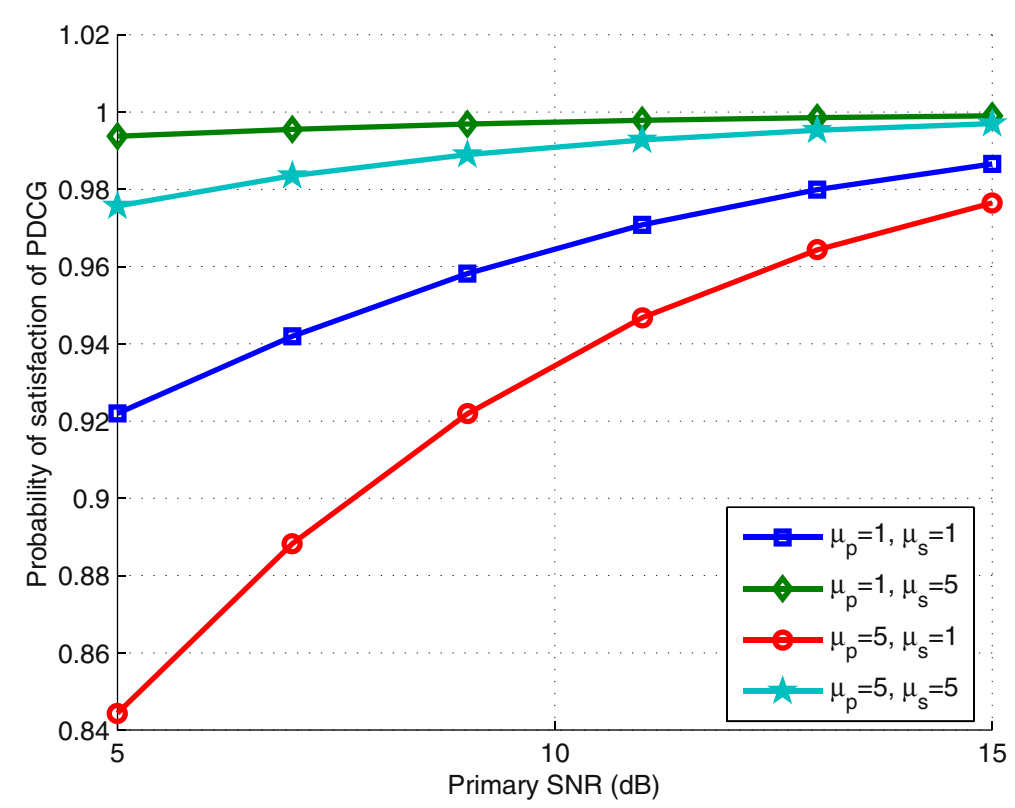

Figure 12 Probability of finding at least one primary user that satisfies the PDCG. 
the performance of rate splitting in th e Gaussian channel where it has been found that rate splitting by the secondary user is useless when the channel between the secondary transmitter and the primary receiver supports larger rate than the channel between the two secondary nodes. Furthermore, on decoding the signal of a primary transmitter at the secondary receiver, a necessary and sufficient condition has been provided to allow the secondary user to decode the primary signal without reducing the range of achievable primary sum rates; in fact, it can only increase the range of achievable secondary rates. Finally, we have shown numerically that the probability of finding at least one primary user that satisfies this condition increases with the signal-to-noise ratio of the primary users.

\section{Appendix 1 - Proof of Theorem 1}

It is sufficient to show that there exists at least one code for which if the rate tuple $\left(R_{1}, R_{2}, S, T\right)$ satisfies (13) to (22), then the rate tuple is achievable. We use the following random code.

\section{Random code generation}

A random code $\mathcal{C}$ is generated as follows. Let $\mathbf{q}=$ $\left(q^{(1)}, \ldots, q^{(n)}\right)$ be a random i.i.d sequence of $\mathcal{Q}^{n}, \mathbf{u}_{k}=$ $\left(u_{k}^{(1)}, \cdots, u_{k}^{(n)}\right), k \in \mathcal{L}_{s}$ a sequence of random variables of $\mathcal{U}^{n}$ that are i.i.d given q. Moreover, $\mathbf{u}_{k}$ and $\mathbf{u}_{k^{\prime}}$ are independent $\forall k \neq k^{\prime}, k, k^{\prime} \in \mathcal{L}_{s}$. Similarly, generate $\mathbf{w}_{l}, l \in \mathcal{N}_{s}$, $\mathbf{x}_{1 i}, i \in \mathcal{M}_{1}$ and $\mathbf{x}_{2 j}, j \in \mathcal{M}_{2}$, which are respectively i.i.d. given $\mathbf{q}$

\section{Encoding}

For primary user 1 to send a message $i \in \mathcal{M}_{1}$, it sends $\mathbf{x}_{1 i}$. Similarly, for primary user 2 to send a message $j \in \mathcal{M}_{2}$, it sends $\mathbf{x}_{2 j}$. For the secondary user to send a message $k l \in \mathcal{L}_{s} \times \mathcal{N}_{s}$, it sends $f^{n}\left(\mathbf{u}_{k} \mathbf{w}_{l} \mid \mathbf{q}\right)=$ $\left(f^{(1)}\left(u_{k}^{(1)} w_{l}^{(1)} \mid q^{(1)}\right), \cdots, f^{(n)}\left(u_{k}^{(n)} w_{l}^{(n)} \mid q^{(n)}\right)\right)$, where $\mathbf{q}$ is known at the transmitters.

\section{Decoding: jointly typical decoding}

We use the concept of jointly typical sequences and the properties of typical sets introduced in Chapter 15 of [18] to implement the decoding functions. Let $A_{\epsilon}^{(n)}$ denote the set of typical $\left(\mathbf{q}, \mathbf{x}_{1}, \mathbf{x}_{2}, \mathbf{w}_{l}, \mathbf{y}_{p}\right)$ sequences, then the primary receiver decides $i j l$ if $\left(\mathbf{q}, \mathbf{x}_{1 i}, \mathbf{x}_{2 j}, \mathbf{w}_{l}, \mathbf{y}_{p}\right) \in A_{\epsilon}^{(n)}$. Also, let $B_{\epsilon}^{(n)}$ denote the set of typical $\left(\mathbf{q}, \mathbf{u}, \mathbf{w}, \mathbf{y}_{s}\right)$ sequences, then secondary receiver decides $k l$ if $\left(\mathbf{q}, \mathbf{u}_{k}, \mathbf{w}_{l}, \mathbf{y}_{s}\right) \in B_{\epsilon}^{(n)}$.

\section{Probability of error analysis}

By the symmetry of the random code generation, the conditional probability of error does not depend on the transmitted messages. Hence, the conditional probability of error is the same as the average probability of error. So, let $i j k l=1111$ be sent. An error occurs if the transmitted codewords are not typical with the received sequences.

\section{For the primary receiver}

Let the event

$$
E_{p}(i j l)=\left\{\left(\mathbf{q}, \mathbf{x}_{1 i}, \mathbf{x}_{2 j}, \mathbf{w}_{l}, \mathbf{y}_{p}\right) \in A_{\epsilon}^{(n)}\right\} ;
$$

hence, the probability of error averaged over the random code $\mathcal{C}$ is

$$
\bar{P} e_{p}^{o}=P\left(E_{p}^{c}(111) \bigcup \cup_{i j l \neq 111} E_{p}(i j l)\right),
$$

where $E_{p}^{c}(111)$ denotes the complement of $E_{p}(111)$. Using union bound, we have

$$
\begin{aligned}
\bar{P} e_{p}^{o} \leq & P\left(E_{p}^{c}(111)\right)+P\left(\cup_{i j l \neq 111} E_{p}(i j l)\right) \\
\leq & P\left(E_{p}^{c}(111)\right)+\left(M_{1}-1\right) P\left(E_{p}(211)\right) \\
& +\left(M_{2}-1\right) P\left(E_{p}(121)\right)+\left(N_{s}-1\right) P\left(E_{p}(112)\right) \\
& +\left(M_{1}-1\right)\left(M_{2}-1\right) P\left(E_{p}(221)\right) \\
& +\left(M_{1}-1\right)\left(N_{s}-1\right) P\left(E_{p}(212)\right) \\
& +\left(M_{2}-1\right)\left(N_{s}-1\right) P\left(E_{p}(122)\right) \\
& +\left(M_{1}-1\right)\left(M_{2}-1\right)\left(N_{s}-1\right) P\left(E_{p}(222)\right) .
\end{aligned}
$$

From the properties of jointly typical sequences [18], $P\left(E_{p}^{c}(111)\right) \rightarrow 0$ as $n \rightarrow \infty$, and

$$
\begin{aligned}
P\left(E_{p}(211)\right) & =2^{-n\left(H\left(X_{1} \mid Q\right)-H\left(X_{1} \mid X_{2} W Y_{p} Q\right)\right)+6 \epsilon} \\
& =2^{-n\left(I\left(X_{1} ; X_{2} W Y_{p} \mid Q\right)\right)+6 \epsilon} \\
& =2^{-n\left(I\left(Y_{p} ; X_{1} \mid W X_{2} Q\right)\right)+6 \epsilon}
\end{aligned}
$$

where the last equality holds from the assumption that $X_{1}$, $X_{2}, U$, and $W$ are conditionally independent given $Q$. Similarly, for other $E_{p}(i j l \neq 111)$ and applying Equations (6) to $(8)$, we get

$$
\begin{aligned}
\bar{P} e_{p}^{o} \leq & 2^{-n\left(I\left(Y_{p} ; X_{1} \mid W X_{2} Q\right)-R_{1}+\eta-6 \epsilon\right)} \\
& +2^{-n\left(I\left(Y_{p} ; X_{2} \mid W X_{1} Q\right)-R_{2}+\eta-6 \epsilon\right)} \\
& +2^{-n\left(I\left(Y_{p} ; W \mid X_{1} X_{2} Q\right)-T+\eta-6 \epsilon\right)} \\
& +2^{-n\left(I\left(Y_{p} ; X_{1} X_{2} \mid W Q\right)-\left(R_{1}+R_{2}\right)+\eta-6 \epsilon\right)} \\
& +2^{-n\left(I\left(Y_{p} ; W X_{1} \mid X_{2} Q\right)-\left(T+R_{1}\right)+\eta-6 \epsilon\right)} \\
& +2^{-n\left(I\left(Y_{p} ; W X_{2} \mid X_{1} Q\right)-\left(T+R_{2}\right)+\eta-6 \epsilon\right)} \\
& +2^{-n\left(I\left(Y_{p} ; X_{1} X_{2} W \mid Q\right)-\left(T+R_{1}+R_{2}\right)+\eta-6 \epsilon\right)} .
\end{aligned}
$$

Thus, if (13) to (19) are satisfied, $\bar{P} e_{p}^{o} \rightarrow \epsilon$ as $n \rightarrow \infty$.

\section{For the secondary receiver}

Let the event

$$
E_{s}(k l)=\left\{\left(\mathbf{q}, \mathbf{u}_{k}, \mathbf{w}_{l}, \mathbf{y}_{s}\right) \in B_{\epsilon}^{(n)}\right\} ;
$$


hence, the probability of decoding error averaged over the random code $\mathcal{C}$ is

$$
\bar{P} e_{s}^{o}=P\left(E_{s}^{c}(11) \bigcup \cup_{k l \neq 11} E_{p}(k l)\right),
$$

where $E_{s}^{c}(11)$ denotes the complement of $E_{s}(11)$. Using union bound, we have

$$
\begin{aligned}
\bar{P} e_{s}^{o} \leq & P\left(E_{s}^{c}(11)\right)+\left(L_{s}-1\right) P\left(E_{s}(21)\right) \\
& +\left(N_{s}-1\right) P\left(E_{s}(12)\right) \\
& +\left(L_{s}-1\right)\left(N_{s}-1\right) P\left(E_{s}(22)\right) .
\end{aligned}
$$

Since $P\left(E_{s}^{c}(11)\right) \rightarrow \epsilon$ as $n \rightarrow \infty$, then

$$
\begin{aligned}
\bar{P} e_{s}^{o} \leq & 2^{-n\left(I\left(Y_{s} ; U \mid W Q\right)-S+\eta-6 \epsilon\right)} \\
& +2^{-n\left(I\left(Y_{s} ; W \mid U Q\right)-T+\eta-6 \epsilon\right)} \\
& +2^{-n\left(I\left(Y_{s} ; U W \mid Q\right)-(S+T)+\eta-6 \epsilon\right)} .
\end{aligned}
$$

So, if (20) to (22) are satisfied, $\bar{P} e_{s}^{o} \rightarrow \epsilon$ as $n \rightarrow \infty$.

This concludes the proof.

\section{Appendix 2 - Proof of Theorem 5}

\section{Sufficiency part}

Suppose (82) is satisfied, we use Figure 5 to prove that $\mathcal{R}^{o}(Z) \subseteq \mathcal{R}_{i}^{r}(Z)$. It is sufficient to show that $R_{p}^{A^{o}}=R_{p}^{A^{r}}$, $R_{s}^{B^{o}} \leq R_{s}^{B^{r}}, R_{s}^{D^{o}} \leq R_{s}^{F^{r}}$ and that lines $2 R_{s}+R_{p}=\rho_{2 p}^{r}$ and $R_{s}+R_{p}=\rho_{s p}^{o}$ intersect at a point $\left(R_{s}^{*}, R_{p}^{*}\right)$ for which $R_{s}^{*} \geq R_{s}^{D^{o}}$, i.e., the intersection between the two lines is outside $\mathcal{R}^{o}(Z)$. Consider that the primary user whose signal is not decodable at the secondary receiver is indexed by $j, j \in\{1,2\}$ and $i \neq j$.

\section{Proof of $R_{p}^{A^{o}}=R_{p}^{A^{r}}$}

From the analysis of the channels $C_{R S}$ and $C_{R S}^{p}$ in Section 3, we have

$$
\begin{aligned}
& R_{p}^{A^{o}}=I\left(Y_{p} ; X_{1} X_{2} \mid W Q\right), \\
& R_{p}^{A^{r}}=I\left(Y_{p} ; X_{j} \mid W X_{i} Q\right)+\sigma_{p}^{*} .
\end{aligned}
$$

From (82), $\sigma_{p}^{*}=I\left(Y_{p}, X_{i} \mid W Q\right)$. Therefore,

$$
R_{p}^{A^{r}}=I\left(Y_{p} ; X_{1} X_{2} \mid W Q\right)=R_{p}^{A^{o}} .
$$

\section{Proof of $R_{s}^{B^{o}} \leq R_{s}^{B^{r}}$}

From the proof of Theorem 2,

$$
R_{s}^{B^{o}}=I\left(Y_{s} ; U \mid W Q\right)+\min \{\overbrace{I\left(Y_{p} ; W \mid Q\right)}^{o_{1}}, \overbrace{I\left(Y_{s} ; W \mid Q\right)}^{o_{2}}\},
$$

and from the proof of Theorem 4,

$$
\begin{aligned}
R_{s}^{B^{r}}= & I\left(Y_{s} ; U \mid W X_{i} Q\right)-\left[I\left(Y_{p} ; X_{i} \mid W Q\right)\right. \\
& \left.-I\left(Y_{s} ; X_{i} \mid W Q\right)\right]^{+}+\min \left\{I\left(Y_{p} ; W \mid Q\right),\right. \\
& I\left(Y_{s} ; W \mid Q\right)+\left[I\left(Y_{s} ; X_{i} \mid W Q\right)-I\left(Y_{p} ; X_{i} \mid W Q\right)\right]^{+}, \\
& \left.I\left(Y_{s} ; W \mid X_{i} Q\right)\right\} .
\end{aligned}
$$

Case I: $\quad$ If $I\left(Y_{p} ; X_{i} \mid W Q\right) \leq I\left(Y_{s} ; X_{i} \mid W Q\right)$

$$
\begin{aligned}
R_{s}^{B^{r}}= & I\left(Y_{s} ; U \mid W X_{i} Q\right)+\min \{\overbrace{I\left(Y_{p} ; W \mid Q\right)}^{v_{1}}, \\
& \overbrace{I\left(Y_{s} ; W \mid Q\right)+I\left(Y_{s} ; X_{i} \mid W Q\right)-I\left(Y_{p} ; X_{i} \mid W Q\right)}^{\nu_{2}}, \\
& \overbrace{I\left(Y_{s} ; W \mid X_{i} Q\right)}^{\nu_{3}}\} .
\end{aligned}
$$

Note that, $v_{1}=o_{1}$.

- If $o_{1} \leq o_{2}$ in (102)

$$
\begin{aligned}
R_{s}^{B^{o}} & =I\left(Y_{s} ; U \mid W Q\right)+I\left(Y_{p} ; W \mid Q\right), \\
R_{s}^{B^{r}} & =I\left(Y_{s} ; U \mid W X_{i} Q\right)+I(Y p ; W \mid Q) \\
& \geq R_{s}^{B^{o}} .
\end{aligned}
$$

- If $o_{2} \leq o_{1}$ in (102)

$$
\begin{aligned}
R_{s}^{B^{o}} & =I\left(Y_{s} ; U \mid W Q\right)+I\left(Y_{s} ; W \mid Q\right) \\
& =I\left(Y_{s} ; U W \mid Q\right) .
\end{aligned}
$$

When $v_{1}=\min \left\{v_{1}, v_{2}, v_{3}\right\}$ in (103), then

$$
\begin{aligned}
R_{s}^{B^{r}} & =I\left(Y_{s} ; U \mid W X_{i} Q\right)+\overbrace{I\left(Y_{p} ; W \mid Q\right)}^{\geq o_{2}} \\
& \geq R_{s}^{B^{o}} .
\end{aligned}
$$

When $v_{2}=\min \left\{v_{1}, v_{2}, v_{3}\right\}$ in (103), then

$$
\begin{aligned}
R_{s}^{B^{r}}= & I\left(Y_{s} ; U \mid W X_{i} Q\right)+I\left(Y_{s} ; W \mid Q\right)+I\left(Y_{s} ; X_{i} \mid W Q\right) \\
& -I\left(Y_{p} ; X_{i} \mid W Q\right) \\
\geq & I\left(Y_{s} ; U \mid W X_{i} Q\right)+I\left(Y_{s} ; W \mid Q\right) \\
\geq & R_{s}^{B^{o}} .
\end{aligned}
$$

When $v_{3}=\min \left\{v_{1}, v_{2}, v_{3}\right\}$ in (103), then

$$
\begin{aligned}
R_{s}^{B^{r}} & =I\left(Y_{s} ; U \mid W X_{i} Q\right)+I\left(Y_{s} ; W \mid X_{i} Q\right) \\
& =I\left(Y_{s} ; U W \mid X_{i} Q\right) \\
& \geq R_{s}^{B^{o}} .
\end{aligned}
$$


Case II: If $I\left(Y_{s} ; X_{i} \mid W Q\right) \leq I\left(Y_{p} ; X_{i} \mid W Q\right)$

$$
\begin{aligned}
R_{s}^{B^{r}}= & I\left(Y_{s} ; U \mid W X_{i} Q\right)+I\left(Y_{s} ; X_{i} \mid W Q\right)-I\left(Y_{p} ; X_{i} \mid W Q\right) \\
& +\min \{\overbrace{I\left(Y_{p} ; W \mid Q\right)}^{v_{4}}, \overbrace{I\left(Y_{s} ; W \mid Q\right)}^{\nu_{5}}\} .
\end{aligned}
$$

Note that $o_{1}=v_{4}$ and $o_{2}=v_{5}$.

- If $o_{1} \leq o_{2}$ in (102)

$$
\begin{aligned}
R_{s}^{B^{o}} & =I\left(Y_{s} ; U \mid W Q\right)+I\left(Y_{p} ; W \mid Q\right), \\
R_{s}^{B^{r}}= & I\left(Y_{s} ; U X_{i} \mid W Q\right)-I\left(Y_{p} ; X_{i} \mid W Q\right)+I\left(Y_{p} ; W \mid Q\right) \\
= & I\left(Y_{s} ; U \mid W Q\right)+I\left(Y_{p} ; W \mid Q\right) \\
& +\overbrace{I\left(Y_{s} ; X_{i} \mid U W Q\right)-I\left(Y_{p} ; X_{i} \mid W Q\right)}^{\geq 0 \text { from }(82)} \\
\geq & R_{s}^{B^{o}} .
\end{aligned}
$$

- If $o_{2} \leq o_{1}$ in (102)

The proof follows exactly as the case of $o_{1} \leq o_{2}$.

$$
\begin{aligned}
\text { Proof of } \boldsymbol{R}_{\boldsymbol{s}}^{\boldsymbol{F}^{r}} \geq \boldsymbol{R}_{\boldsymbol{s}}^{\boldsymbol{D}^{o}} & \begin{aligned}
R_{s}^{F^{r}}= & I\left(Y_{s} ; U \mid W X_{i} Q\right)+\min \left\{I\left(Y_{s} ; W \mid X_{i} Q\right),\right. \\
& \left.I\left(Y_{p} ; W \mid X_{1} X_{2} Q\right)\right\} . \\
R_{s}^{D^{o}}= & I\left(Y_{s} ; U \mid W Q\right)+\min \left\{I\left(Y_{s} ; W \mid Q\right),\right. \\
& \left.I\left(Y_{p} ; W \mid X_{1} X_{2} Q\right)\right\} .
\end{aligned}
\end{aligned}
$$

It is obvious that each term in $R_{S}^{F^{r}}$ is greater than or equal to its corresponding term in $R_{s}^{D^{o}}$. Hence, $R_{s}^{F^{r}} \geq R_{s}^{D^{o}}$.

\section{Proof of the intersection point between the two lines} $2 R_{s}+R_{p}=\rho_{2 p}^{r}$ and $R_{s}+R_{p}=\rho_{s p}^{o}$ occurs at a point $\left(R_{s}^{*}, R_{p}^{*}\right)$ where $R_{s}^{*} \geq R_{s}^{D^{o}}$

The secondary rate of the intersection point is $R_{s}^{*}=\rho_{2 p}^{r}-$ $\rho_{s p}^{o}$. From Theorems 2 and 4 ,

$$
\begin{aligned}
R_{s}^{D^{o}}= & I\left(Y_{s} ; U \mid W Q\right)+\sigma^{*}, \\
R_{s}^{*}= & 2 I\left(Y_{s} ; U \mid W X_{i} Q\right)+2 \sigma_{s}^{*}+I\left(Y_{p} ; X_{j} \mid W X_{i} Q\right) \\
& -\left[\sigma_{s}^{*}-I\left(Y_{p} ; W \mid X_{i} Q\right)\right]^{+}+\min \left\{I\left(Y_{s} ; X_{i} \mid W Q\right),\right. \\
& I\left(Y_{s} ; W X_{i} \mid Q\right)-\sigma_{s}^{*}, I\left(Y_{p} ; X_{i} \mid Q\right)+\left[I\left(Y_{p} ; W \mid X_{i} Q\right)\right. \\
& \left.\left.-\sigma_{s}^{*}\right]^{+}, I\left(Y_{p} ; X_{i} \mid W Q\right)\right\}-I\left(Y_{p} ; X_{1} X_{2} \mid W Q\right) \\
& -I\left(Y_{s} ; U \mid W Q\right)-\min \left\{I\left(Y_{s} ; W \mid Q\right), I\left(Y_{p} ; W \mid Q\right)\right\} .
\end{aligned}
$$

Hence, it is required to show that $R_{s}^{*} \geq R_{s}^{D^{o}}$.

$$
\text { If } \sigma_{s}^{*}=I\left(Y_{s} ; W \mid X_{i} Q\right) \leq I\left(Y_{p} ; W \mid X_{1} X_{2} Q\right)
$$

- If $I\left(Y_{s} ; W \mid X_{i} Q\right) \leq I\left(Y_{p} ; W \mid X_{i} Q\right)$, from (105) and (106), we have

$$
\begin{aligned}
R_{s}^{D^{o}}= & I\left(Y_{s} ; U \mid W Q\right)+I\left(Y_{s} ; W \mid Q\right)=I\left(Y_{s} ; U W \mid Q\right), \quad(107) \\
R_{s}^{*}= & 2 I\left(Y_{s} ; U \mid W X_{i} Q\right)+2 I\left(Y_{s} ; W \mid X_{i} Q\right)-I\left(Y_{s} ; U \mid W Q\right) \\
& +I\left(Y_{p} ; X_{j} \mid W X_{i} Q\right)+\min \{\overbrace{I\left(Y_{s} ; X_{i} \mid Q\right)}^{v_{6}}, \\
& \overbrace{I\left(Y_{p} ; X_{i} \mid Q\right)+I\left(Y_{p} ; W \mid X_{i} Q\right)-I\left(Y_{s} ; W \mid X_{i} Q\right)}^{v_{7}}, \\
& \overbrace{I\left(Y_{p} ; X_{i} \mid W Q\right)}^{v_{8}}\}-I\left(Y_{p} ; X_{1} X_{2} \mid W Q\right)-\min \left\{v_{4}, \nu_{5}\right\} .
\end{aligned}
$$

When $v_{6}=\min \left\{v_{6}, v_{7}, v_{8}\right\}$ in (108), then

$$
\begin{aligned}
R_{s}^{*}= & I\left(Y_{s} ; U \mid W X_{i} Q\right)-I\left(Y_{s} ; U \mid W Q\right)+\overbrace{I\left(Y_{s} ; U W \mid Q\right)}^{=R_{s}^{D^{o}}} \\
& +I\left(Y_{s} ; W \mid X_{i} Q\right)-\min \left\{v_{4}, \nu_{5}\right\}+I\left(Y_{p} ; X_{j} \mid W X_{i} Q\right) \\
& \geq I\left(Y_{p} ; X_{i} \mid W Q\right) \text { from }(82) \\
& +\overbrace{I\left(Y_{s} ; X_{i} \mid U W Q\right)}-I\left(Y_{p} ; X_{1} X_{2} \mid W Q\right) \\
\geq & R_{s}^{D^{o}} .
\end{aligned}
$$

When $v_{7}=\min \left\{v_{6}, v_{7}, v_{8}\right\}$ in (108), then

$$
\begin{aligned}
R_{s}^{*}= & I\left(Y_{s} ; U \mid W X_{i} Q\right)-I\left(Y_{s} ; U \mid W Q\right)+\overbrace{I\left(Y_{s} ; U W \mid X_{i} Q\right)}^{\geq R_{s}^{D^{o}}} \\
& +v_{4}-\min \left\{v_{4}, v_{5}\right\} \\
\geq & R_{s}^{D^{o}} .
\end{aligned}
$$

When $v_{8}=\min \left\{v_{6}, v_{7}, v_{8}\right\}$ in (108), then

$$
\begin{aligned}
R_{s}^{*}= & I\left(Y_{s} ; U \mid W X_{i} Q\right)-I\left(Y_{s} ; U \mid W Q\right)+\overbrace{I\left(Y_{s} ; U W \mid X_{i} Q\right)}^{\geq R_{s}^{D^{o}}} \\
& +I\left(Y_{s} ; W \mid X_{i} Q\right)-\min \left\{v_{4}, \nu_{5}\right\} \\
\geq & R_{s}^{D^{o}} .
\end{aligned}
$$

- If $I\left(Y_{s} ; W \mid X_{i} Q\right) \geq I\left(Y_{p} ; W \mid X_{i} Q\right)$,

$R_{s}^{D^{o}}$ will remain the same as (107) and $R_{s}^{*}$ will be given by

$$
\begin{aligned}
R_{s}^{*}= & 2 I\left(Y_{s} ; U \mid W X_{i} Q\right)+2 I\left(Y_{s} ; W \mid X_{i} Q\right)-I\left(Y_{s} ; U \mid W Q\right) \\
& +I\left(Y_{p} ; X_{j} \mid W X_{i} Q\right)+I\left(Y_{p} ; W \mid X_{i} Q\right) \\
& -I\left(Y_{s} ; W \mid X_{i} Q\right)+\min \{\overbrace{I\left(Y_{s} ; X_{i} \mid Q\right)}^{v_{9}} \overbrace{I\left(Y_{p} ; X_{i} \mid Q\right)}^{v_{10}}\} \\
& -I\left(Y_{p} ; X_{1} X_{2} \mid W Q\right)-\min \left\{v_{4}, v_{5}\right\} .
\end{aligned}
$$


When $v_{9}=\min \left\{v_{9}, v_{10}\right\}$ in (109), then

$$
\begin{aligned}
R_{s}^{*}= & I\left(Y_{s} ; U \mid W X_{i} Q\right)-I\left(Y_{s} ; U \mid W Q\right)+\overbrace{I\left(Y_{s} ; U W \mid Q\right)}^{=R_{s}^{D^{o}}} \\
& +I\left(Y_{p} ; W \mid X_{i} Q\right)-\min \left\{v_{4}, v_{5}\right\}+I\left(Y_{s} ; X_{i} \mid U W Q\right) \\
& +I\left(Y_{p} ; X_{j} \mid W X_{i} Q\right)-I\left(Y_{p} ; X_{1} X_{2} \mid W Q\right) \\
\geq & R_{s}^{D^{o}} .
\end{aligned}
$$

When $v_{10}=\min \left\{v_{9}, v_{10}\right\}$ in (109), then

$$
\begin{aligned}
R_{s}^{*}= & I\left(Y_{s} ; U \mid W X_{i} Q\right)-I\left(Y_{s} ; U \mid W Q\right)+\overbrace{I\left(Y_{s} ; U W \mid X_{i} Q\right)}^{\geq R_{s}^{D^{o}}} \\
& +I\left(Y_{p} ; W \mid X_{i} Q\right)-\min \left\{v_{4}, v_{5}\right\} \\
\geq & R_{s}^{D^{o}}
\end{aligned}
$$

If $\sigma_{s}^{*}=I\left(Y_{p} ; W \mid X_{1} X_{2} Q\right) \leq I\left(Y_{s} ; W \mid X_{i} Q\right)$ from and (106), we have

$$
R_{s}^{D^{o}}=I\left(Y_{s} ; U \mid W Q\right)+\min \{\overbrace{I\left(Y_{s} ; W \mid Q\right)}^{o_{2}}, \overbrace{I\left(Y_{p} ; W \mid X_{1} X_{2} Q\right)}^{o_{3}}\},
$$

$$
\begin{aligned}
R_{s}^{*}= & 2 I\left(Y_{s} ; U \mid W X_{i} Q\right)-I\left(Y_{s} ; U \mid W Q\right)+I\left(Y_{p} ; W \mid X_{i} Q\right) \\
& +I\left(Y_{p} ; X_{j} \mid W X_{i} Q\right)+I\left(Y_{p} ; W \mid X_{1} X_{2} Q\right) \\
& +\min \{\overbrace{I\left(Y_{p} ; X_{i} \mid Q\right)}^{v_{10}}, \overbrace{I\left(Y_{s} ; X_{i} \mid W Q\right)}^{v_{11}}, \\
& \overbrace{I\left(Y_{s} ; W X_{i} \mid Q\right)-I\left(Y_{p} ; W \mid X_{1} X_{2} Q\right)}^{v_{12}}\}-\min \left\{v_{4}, v_{5}\right\} \\
& -I\left(Y_{p} ; X_{1} X_{2} \mid W Q\right)
\end{aligned}
$$

- If $o_{2} \leq o_{3}$ in (110),

$$
R_{s}^{D^{o}}=I\left(Y_{s} ; U \mid W Q\right)+I\left(Y_{s} ; W \mid Q\right)=I\left(Y_{s} ; U W \mid Q\right) .
$$

When $v_{10}=\min \left\{v_{10}, v_{11}, v_{12}\right\}$ in $(111)$, then

$$
\begin{aligned}
R_{s}^{*}= & I\left(Y_{s} ; U \mid W X_{i} Q\right)-I\left(Y_{s} ; U \mid W Q\right)+v_{4} \\
& -\min \left\{v_{4}, \nu_{5}\right\}+\overbrace{I\left(Y_{s} ; U \mid W X_{i} Q\right)+o_{3}}^{\geq R_{s}^{D^{o}}} .
\end{aligned}
$$

Since $o_{2} \leq o_{3}$, then $v_{11}$ cannot be smaller than $v_{12}$. When $v_{12}=\min \left\{v_{10}, v_{11}, v_{12}\right\}$, then

$$
\begin{aligned}
R_{s}^{*}= & I\left(Y_{s} ; U \mid W X_{i} Q\right)-I\left(Y_{s} ; U \mid W Q\right)+I\left(Y_{p} ; W \mid X_{i} Q\right) \\
& -\min \left\{v_{4}, v_{5}\right\}+\overbrace{I\left(Y_{s} ; U W \mid Q\right)}^{=R_{s}^{D^{o}}}+I\left(Y_{s} ; X_{i} \mid U W Q\right) \\
& +I\left(Y_{p} ; X_{j} \mid W X_{i} Q\right)-I\left(Y_{p} ; X_{1} X_{2} \mid W Q\right) \\
\geq & R_{s}^{D^{o}} .
\end{aligned}
$$

- If $o_{2} \geq o_{3}$

$$
R_{s}^{D^{o}}=I\left(Y_{s} ; U \mid W Q\right)+I\left(Y_{p} ; W \mid X_{1} X_{2} Q\right) .
$$

When $v_{10}=\min \left\{v_{10}, v_{11}, v_{12}\right\}$ in (111), then

$$
\begin{aligned}
R_{s}^{*}= & I\left(Y_{s} ; U \mid W X_{i} Q\right)-I\left(Y_{s} ; U \mid W Q\right) \\
& +\overbrace{I\left(Y_{s} ; U \mid W X_{i} Q\right)+I\left(Y_{p} ; W \mid X_{1} X_{2} Q\right)}^{\geq R_{s}^{D^{o}}} \\
\geq & R_{s}^{D^{o}} .
\end{aligned}
$$

When $v_{11}=\min \left\{v_{10}, v_{11}, v_{12}\right\}$ in (111), then

$$
\begin{aligned}
R_{s}^{*}= & I\left(Y_{s} ; U \mid W X_{i} Q\right)-I\left(Y_{s} ; U \mid W Q\right)+I\left(Y_{s} ; X_{i} \mid U W Q\right) \\
& +I\left(Y_{p} ; X_{j} \mid W X_{i} Q\right)-I\left(Y_{p} ; X_{1} X_{2} \mid W Q\right)-v_{4} \\
& +I\left(Y_{p} ; W \mid X_{i} Q\right)+\overbrace{I\left(Y_{s} ; U \mid W Q\right)+I\left(Y_{p} ; W \mid X_{1} X_{2} Q\right)}^{=R_{s}^{D^{o}}} \\
\geq & R_{s}^{D^{o}} .
\end{aligned}
$$

Since $o_{2} \geq o_{3}$, then $v_{12}$ cannot be smaller than $v_{11}$.

\section{Necessity part}

Suppose $\mathcal{R}^{o}(Z) \subseteq \mathcal{R}_{i}^{r}(Z)$ then $R_{p}^{A^{o}}$ must be not larger than $R_{p}^{A^{r}}$ which necessitates the satisfaction of (82).

This concludes the proof.

\section{Appendix 3 - Proof of Theorem 6}

From the definition of $\delta^{\prime o}(Z)$ and $\delta_{1}^{\prime r}(Z)$, it is clear that $\delta^{o}(Z) \subseteq \delta^{\prime o}(Z)$ and $\delta_{1}^{r}(Z) \subseteq \delta_{1}^{\prime r}(Z)$. Consequently, $\mathcal{R}^{o}(Z) \subseteq \mathcal{R}^{\prime o}(Z), \mathcal{R}_{1}^{r} \subseteq \mathcal{R}_{1}^{\prime r}(Z)$, and $\mathcal{R}_{1}^{r}(Z) \subseteq \mathcal{R}_{1}^{\prime r}(Z)$. However, we show that if there exists $Z \in \mathcal{P}^{*}$ such that a rate tuple $\left(R_{s}, R_{p}\right)$ belongs to $\mathcal{R}^{\prime o}(Z)$ but does not belong to $\mathcal{R}^{o}(Z)$, then there exists another $Z^{\prime} \in \mathcal{P}^{*}$ for which $\left(R_{s}, R_{p}\right)$ belongs to $\mathcal{R}^{o}\left(Z^{\prime}\right)$. And we do the same for $\mathcal{R}_{1}^{\prime r}(Z)$, $\mathcal{R}_{1}^{r}(Z)$.

\section{For $\mathcal{R}^{o}(Z)$}

Following a similar procedure to that used in the proof of Theorem 2 , the region $\mathcal{R}^{\prime o}(Z)$ is defined by

$$
\begin{aligned}
R_{p} \leq & I\left(Y_{p} ; X_{1} X_{2} \mid W Q\right), \\
R_{s} \leq & I\left(Y_{s} ; U \mid W Q\right)+\min \left\{I\left(Y_{s} ; W \mid Q\right),\right. \\
& \left.I\left(Y_{p} ; W X_{1} \mid X_{2} Q\right), I\left(Y_{p} ; W X_{2} \mid X_{1} Q\right)\right\}, \\
R_{s}+R_{p} \leq & I\left(Y_{s} ; U \mid W Q\right)+I\left(Y_{p} ; X_{1} X_{2} \mid W Q\right) \\
& +\min \left\{I\left(Y_{s} ; W \mid Q\right), I\left(Y_{p} ; W \mid Q\right)\right\} .
\end{aligned}
$$

Suppose that at a certain $Z \in \mathcal{P}^{*}, R_{s}^{\prime}>I\left(Y_{s} ; U \mid W Q\right)+$ $I\left(Y_{p} ; W \mid X_{1} X_{2} Q\right)$, hence, the rate tuple $\left(R_{s}^{\prime}, R_{p}^{\prime}\right) \in \mathcal{R}^{\prime o}(Z)$ but $\left(R_{s}^{\prime}, R_{p}^{\prime}\right) \notin \mathcal{R}^{o}(Z)$. From (112) to (114), $\left(R_{s}^{\prime}, R_{p}^{\prime}\right)$ has to satisfy

$$
\begin{aligned}
R_{s} \leq I\left(Y_{s} ; U W \mid Q\right) & =I\left(Y_{s} ; X_{s} \mid Q\right), \\
R_{p} & <I\left(Y_{p} ; X_{1} X_{2} \mid Q\right) .
\end{aligned}
$$


Now, assume another $Z^{\prime} \in \mathcal{P}^{*}$ such that $W=\phi$, i.e., no rate splitting. At this $Z^{\prime}, \mathcal{R}^{o}\left(Z^{\prime}\right)$ is given by

$$
\begin{aligned}
& R_{s} \leq I\left(Y_{s} ; X_{s} \mid Q\right), \\
& R_{p} \leq I\left(Y_{p} ; X_{1} X_{2} \mid Q\right) .
\end{aligned}
$$

Then it is clear that $\left(R_{s}^{\prime}, R_{p}^{\prime}\right) \in \mathcal{R}^{o}\left(Z^{\prime}\right)$. Thus,

$$
\mathcal{R}^{\prime o}(Z) \subseteq \mathcal{R}^{o}(Z) \cup \mathcal{R}^{o}\left(Z^{\prime}\right)
$$

\section{For $\mathcal{R}_{1}^{\prime r}(Z)$}

First, for a point $\left(R_{s}^{\prime \prime}, R_{p}^{\prime \prime}\right)$ such that $R_{s}^{\prime \prime}>I\left(Y_{s} ; U \mid W Q\right)+$ $I\left(Y_{p} ; W \mid X_{1} X_{2} Q\right)$ at a specific $Z \in \mathcal{P}^{*}$, a similar argument as in the above subsection ('For $\mathcal{R}^{o}(Z)^{\prime}$ ), or in Lemma 2 of [19], can show that there exists $Z^{\prime \prime} \in \mathcal{P}^{*}$ such that $\left(R_{s}^{\prime \prime}, R_{p}^{\prime \prime}\right) \in \mathcal{R}_{1}^{r}\left(Z^{\prime \prime}\right)$.

Second, for another point $\left(R_{s}^{* *}, R_{p}^{* *}\right)$ such that $R_{p}^{* *}>$ $I\left(Y_{p} ; X_{2} \mid W X_{1} Q\right)+I\left(Y_{s} ; X_{1} \mid U W Q\right)$, or in other words $R_{1}^{* *}>I\left(Y_{s} ; X_{1} \mid U W Q\right)$, in this case, $\delta_{1}^{\prime r}(Z) \subset \delta^{\prime o}(Z)$. And since $\mathcal{R}^{\prime o}(Z)$ is the set of $\left(R_{s}, R_{p}\right)$ corresponding to $\delta^{\prime o}(Z)$ for which $R_{s}=S+T$ and $R_{p}=R_{1}+R_{2}$, then $\mathcal{R}_{1}^{\prime r}(Z) \subset$ $\mathcal{R}^{\prime o}(Z)$. Moreover, it has been shown in the above subsection ('For $\left.\mathcal{R}^{o}(Z)^{\prime}\right)$ that $\mathcal{R}^{\prime o}(Z) \subseteq \mathcal{R}^{o}(Z) \cup \mathcal{R}^{o}\left(Z^{\prime}\right)$. Therefore,

$$
\mathcal{R}_{1}^{\prime r}(Z) \subseteq \mathcal{R}_{1}^{r}(Z) \cup \mathcal{R}_{1}^{r}\left(Z^{\prime \prime}\right) \cup \mathcal{R}^{o}(Z) \cup \mathcal{R}^{o}\left(Z^{\prime}\right) .
$$

Consequently,

$$
\mathcal{R}_{1}^{\prime}=\mathcal{R}_{1} \text {. }
$$

\section{Appendix 4 - Proof of Theorem 7}

\section{Sufficiency part}

We refer to Figure 3 to determine the effect of varying $\lambda$ on $\mathcal{R}^{o}(Z)$ where $Z \in \mathcal{G}\left(P_{1}, P_{2}, P_{s}\right)$.

- Point A:

$$
R_{p}^{A}=\rho_{p}^{o}=\tau\left(\frac{g_{1}^{p} P_{1}+g_{2}^{p} P_{2}}{g_{s}^{p} \lambda P_{s}+N_{0}}\right)
$$

- Point D:

$$
R_{s}^{D}=\rho_{s}^{o}=\tau\left(\frac{P_{s} g_{s}^{s}}{g_{1}^{s} P_{1}+g_{2}^{s} P_{2}+N_{0}}\right)
$$

- $R_{s}+R_{p}$ : We can move $\rho_{p}^{o}+I\left(Y_{s} ; U \mid W\right)$ inside the $\min \{\}$ to have

$$
\begin{aligned}
& \rho_{s p}^{o}=\min \left\{\tau\left(\frac{g_{1}^{p} P_{1}+g_{2}^{p} P_{2}}{g_{s}^{p} \lambda P_{s}+N_{0}}\right)+\tau\left(\frac{g_{s}^{s} P_{s}}{P_{1} g_{1}^{s}+P_{2} g_{2}^{s}+N_{0}}\right),\right. \\
& \left.\tau\left(\frac{\lambda P_{s} g_{s}^{s}}{g_{1}^{s} P_{1}+g_{2}^{s} P_{2}+N_{0}}\right)+\tau\left(\frac{g_{1}^{p} P_{1}+g_{2}^{p} P_{2}+\lambda g_{s}^{\bar{p}} P_{s}}{\lambda g_{s}^{p} P_{s}}\right)\right\}+.
\end{aligned}
$$

Clearly, the first argument of the $\min \{$,$\} is monotoni-$ cally decreasing with $\lambda$. By taking the first derivative of the second argument with respect to $\lambda$, it turns out that such second argument is also monotonically decreasing with $\lambda$ if $g_{s}^{s} N_{0} \leq g_{s}^{p}\left(g_{1}^{s} P_{1}+g_{2}^{s} P_{2}+N_{0}\right)$. It is therefore obvious that if (97) is satisfied, $\rho_{s p}^{o}$ increase as $\lambda$ decreases. Consequently, $\mathcal{R}^{o}(Z)$ at $\lambda=0$ includes all other $\mathcal{R}^{o}(Z)$ obtained at $0<\lambda \leq 1$. Hence, $\mathcal{R}^{o}(Z)$ coincides on $\mathcal{R}_{g}^{o}$ at $\lambda=0$.

\section{Necessity part}

Here we prove that the condition in (97) is necessary for $\mathcal{R}^{o}(Z)$ to coincide on $\mathcal{R}_{g}^{o}$ at $\lambda=0$ and $Z \in \mathcal{G}\left(P_{1}, P_{2}, P_{s}\right)$. We do so by showing that if (97) is not satisfied, then for any two different values of $\lambda$, the corresponding rate regions do not contain one another. Assume that (97) is not satisfied, then by referring to Figure 3 we have

- Point A:

$$
R_{p}^{A}=\tau\left(\frac{g_{1}^{p} P_{1}+g_{2}^{p} P_{2}}{g_{s}^{p} \lambda P_{s}+N_{0}}\right)
$$

i.e., the $R_{p}^{A}$ decreases as $\lambda$ increases.

- Point $D$ :

$$
R_{s}^{D}=\tau\left(\frac{g_{s}^{s} \lambda P_{s}}{g_{1}^{s} P_{1}+g_{2}^{s} P_{2}+N_{0}}\right)+\tau\left(\frac{g_{s}^{p} \bar{\lambda} P_{s}}{g_{s}^{p} \lambda P_{s}+N_{0}}\right)
$$

Then by substituting with $\bar{\lambda}=1-\lambda$ and differentiating $R_{s}^{D}$ with respect to $\lambda$, we get

$$
\frac{\partial R_{s}^{D}}{\partial \lambda}=\frac{\frac{1}{2 \ln (2)} P_{s}\left(g_{s}^{s} N_{0}-g_{s}^{p}\left(P_{1} g_{1}^{s}+P_{2} g_{2}^{s}+N_{0}\right)\right)}{\left(\lambda P_{s} g_{s}^{p}+N_{0}\right)\left(P_{1} g_{1}^{s}+P_{2} g_{2}^{s}+\lambda P_{s} g_{s}^{s}+N_{0}\right)},
$$

and since the condition (97) is not satisfied, the numerator of (119) is always positive; therefore, $R_{s}^{D}$ increases as $\lambda$ increases.

Since $R_{p}^{A}$ decreases and $R_{s}^{D}$ increases as $\lambda$ increases, then for any two different values of $\lambda$ the corresponding rate regions will never contain one another. Hence, the overall rate region $\mathcal{R}_{g}^{o}$ does not coincide on a certain $\mathcal{R}^{o}(Z)$ at a certain $\lambda$. This concludes the proof.

\section{Appendix 5 - Proof of Theorem 8}

For the proof, we consider $i=1$, i.e., the secondary user is assumed to be able to decode the signal of primary user 1 .

\section{Sufficiency part}

In this part, we show that if inequality (99) is satisfied, then $\mathcal{R}_{1 g}^{r}$ coincides on $\mathcal{R}_{1}^{r}(Z)$ at $\lambda=0$. We refer to Figure 4 and determine the effect of varying $\lambda$ on $\mathcal{R}_{1}^{r}(Z)$, $Z \in \mathcal{G}\left(P_{1}, P_{2}, P_{s}\right)$ as follows. 
At point A

$$
\begin{aligned}
R_{p}^{r A}= & \tau\left(\frac{g_{2}^{p} P_{2}}{g_{s}^{p} \lambda P_{s}+N_{0}}\right)+\min \left\{\tau\left(\frac{g_{1}^{s} P_{1}}{g_{2}^{s} P_{2}+N_{0}}\right),\right. \\
& \left.\tau\left(\frac{g_{1}^{p} P_{1}}{g_{s}^{p} \lambda P_{s}+g_{2}^{p} P_{2}+N_{0}}\right)\right\} .
\end{aligned}
$$

Therefore, $R_{p}^{r A}$ increases as $\lambda$ decreases.

\section{At point $F$}

$$
R_{s}^{r F}=\tau\left(\frac{g_{s}^{s} P_{s}}{g_{2}^{s} P_{2}+N_{0}}\right)
$$

Hence, $R_{s}^{r F}$ does not depend on $\lambda$.

$$
\begin{aligned}
R_{s}^{r}+ & R_{p}^{r}=\rho_{s p}^{r} \\
\rho_{s p}^{r}= & \tau\left(\frac{g_{s}^{s} \lambda P_{s}}{g_{2}^{s} P_{2}+N_{0}}\right)+\tau\left(\frac{g_{2}^{p} P_{2}}{g_{s}^{p} \lambda P_{s}+N_{0}}\right) \\
& +\min \{\overbrace{\left(\frac{g_{s}^{p} \bar{\lambda} P_{s}+g_{1}^{p} P_{1}}{g_{s}^{p} \lambda P_{s}+g_{2}^{p} P_{2}+N_{0}}\right)}^{\mu_{1}} \overbrace{\left(\frac{g_{s}^{s} \bar{\lambda} P_{s}+g_{1}^{s} P_{1}}{g_{s}^{s} \lambda P_{s}+g_{2}^{s} P_{2}+N_{0}}\right)}^{\mu_{2}}) \\
& \overbrace{\left(\frac{\mu_{3}}{g_{s}^{p} \lambda P_{s}+g_{2}^{p} P_{2}+N_{0}}\right)+\tau\left(\frac{g_{1}^{s} P_{1}}{g_{s}^{s} \lambda P_{s} g_{2}^{s}+P_{2}+N_{0}}\right)}^{\mu_{4}}) \\
& \overbrace{\left(\frac{g_{1}^{p} P_{1}}{g_{s}^{p} \lambda P_{s}+g_{2}^{p} P_{2}+N_{0}}\right)+\tau\left(\frac{g_{s}^{s} \bar{\lambda} P_{s}}{g_{s}^{s} \lambda P_{s}+g_{s}^{s} P_{2}+N_{0}}\right)}^{\mu^{p}})
\end{aligned}
$$

\section{When $\mu_{1}=\min \left\{\mu_{1}, \mu_{2}, \mu_{3}, \mu_{4}\right\}$ in (120)}

$$
\begin{aligned}
\rho_{s p}^{r}= & \tau\left(\frac{g_{s}^{s} \lambda P_{s}}{g_{2}^{s} P_{2}+N_{0}}\right)+\tau\left(\frac{g_{s}^{p} \bar{\lambda} P_{s}+g_{1}^{p} P_{1}+g_{2}^{p} P_{2}}{g_{s}^{p} \lambda P_{s}+N_{0}}\right) . \\
\frac{\partial \rho_{s p}^{r}}{\partial \lambda} & =-\frac{0.5 P_{s}\left(g_{s}^{p} g_{2}^{s} P_{2}+g_{s}^{p} N_{0}-g_{s}^{s} N_{0}\right)}{\ln 2\left(g_{s}^{p} \lambda P_{s}+N_{0}\right)\left(g_{s}^{s} \lambda P_{s}+g_{2}^{s} P_{2}+N_{0}\right)} \\
& \leq 0 \text { from (99). }
\end{aligned}
$$

Hence, $\rho_{s p}^{r}$ decreases with $\lambda$. Note that, $\bar{\lambda}=1-\lambda$.

\section{When $\mu_{2}=\min \left\{\mu_{1}, \mu_{2}, \mu_{3}, \mu_{4}\right\}$ in (120)}

$$
\tau\left(\frac{g_{s}^{s} P_{s}+g_{1}^{s} P_{1}}{g_{2}^{s} P_{2}+N_{0}}\right)+\tau\left(\frac{g_{2}^{p} P_{2}}{g_{s}^{p} \lambda P_{s}+N_{0}}\right),
$$

i.e., $\rho_{s p}^{r}$ decreases with $\lambda$.
When $\mu_{3}=\min \left\{\mu_{1}, \mu_{2}, \mu_{3}, \mu_{4}\right\}$ in (120)

$$
\begin{aligned}
\rho_{s p}^{r} & =\tau\left(\frac{g_{s}^{s} \lambda P_{s}+g_{1}^{s} P_{1}}{g_{s}^{s} P_{2}+N_{0}}\right)+\tau\left(\frac{g_{s}^{p} \bar{\lambda} P_{s}+g_{2}^{p} P_{2}}{g_{s}^{p} \lambda P_{s}+N_{0}}\right) . \\
\frac{\partial \rho_{s p}^{r}}{\partial \lambda} & =-\frac{0.5 P_{s}\left(g_{s}^{p} g_{2}^{s} P_{2}+g_{s}^{p} g_{1}^{s} P_{1}+g_{s}^{p} N_{0}-g_{s}^{s} N_{0}\right)}{\ln 2\left(g_{s}^{p} \lambda P_{s}+N_{0}\right)\left(g_{s}^{s} \lambda P_{s}+g_{2}^{s} P_{2}+g_{1}^{s} P_{1}+N_{0}\right)} \\
& \leq 0 \text { from (99). }
\end{aligned}
$$

Thus, $\rho_{s p}$ decreases with $\lambda$.

When $\mu_{4}=\min \left\{\mu_{1}, \mu_{2}, \mu_{3}, \mu_{4}\right\}$ in (120)

$$
\rho_{s p}^{r}=\tau\left(\frac{g_{s}^{s} P_{s}}{g_{s}^{s} P_{2}+N_{0}}\right)+\tau\left(\frac{g_{1}^{p} P_{1}+g_{2}^{p} P_{2}}{g_{s}^{p} \lambda P_{s}+N_{0}}\right) .
$$

Therefore, $\rho_{s p}^{r}$ decreases with $\lambda$.

$$
\begin{aligned}
& R_{s}^{r}+2 R_{p}^{r}=\rho_{s 2}^{r} \\
& \rho_{s 2}^{r}=2 \tau\left(\frac{g_{2}^{p} P_{2}}{g_{s}^{p} \lambda P_{s}+N_{0}}\right)+2 \sigma_{p}^{*}+\tau\left(\frac{g_{s}^{s} \lambda P_{s}}{g_{2}^{s} P_{2}+N_{0}}\right) \\
&-\left[\sigma_{p}^{*}-\tau\left(\frac{g_{1}^{s} P_{1}}{g_{s}^{s} \lambda P_{s}+g_{2}^{s} P_{2}+N_{0}}\right)\right]^{+}+\min \{ \\
& \tau\left(\frac{g_{s}^{p} \bar{\lambda} P_{s}}{g_{s}^{p} \lambda P_{s}+g_{2}^{p} P_{2}+N_{0}}\right), \tau\left(\frac{g_{s}^{p} \bar{\lambda} P_{s}+g_{1}^{p} P_{1}}{g_{s}^{p} \lambda P_{s}+g_{2}^{p} P_{2}+N_{0}}\right)-\sigma_{p}^{*}, \\
& \tau\left(\frac{g_{s}^{s} \bar{\lambda} P_{s}}{g_{s}^{s} \lambda P_{s}+g_{2}^{s} P_{2}+N_{0}}\right), \tau\left(\frac{g_{s}^{s} \bar{\lambda} P_{s}}{g_{s}^{s} \lambda P_{s}+g_{1}^{p} P_{1}+g_{2}^{p} P_{2}+N_{0}}\right) \\
&+ {\left[\tau\left(\frac{g_{1}^{s} P_{1}}{g_{s}^{s} \lambda P_{s}+g_{2}^{s} P_{2}+N_{0}}\right)-\sigma_{p}^{*}\right], } \\
& \sigma_{p}^{*}= \min \left\{\tau\left(\frac{g_{1}^{p} P_{1}}{g_{s}^{p} \lambda P_{s}+g_{2}^{p} P_{2}+N_{0}}\right), \tau\left(\frac{g_{1}^{s} P_{1}}{g_{2}^{s} P_{2}+N_{0}}\right)\right\} .
\end{aligned}
$$

$$
\begin{aligned}
\text { At } \boldsymbol{\sigma}_{\boldsymbol{p}}^{*} & =\boldsymbol{\tau}\left(\frac{g_{\mathbf{1}}^{\boldsymbol{p}} \boldsymbol{P}_{\mathbf{1}}}{g_{s}^{p} \lambda \boldsymbol{P}_{\boldsymbol{s}}+\boldsymbol{g}_{2}^{\boldsymbol{p}} \boldsymbol{P}_{\mathbf{2}}+\boldsymbol{N}_{\mathbf{0}}}\right) \leq \boldsymbol{\tau}\left(\frac{\boldsymbol{g}_{\mathbf{1}}^{\boldsymbol{s}} \boldsymbol{P}_{\mathbf{1}}}{\boldsymbol{g}_{2}^{\mathbf{s}} \boldsymbol{P}_{\mathbf{2}}+\boldsymbol{N}_{\mathbf{0}}}\right) \\
\rho_{s 2}^{r}= & 2 \tau\left(\frac{g_{1}^{p} P_{1}+g_{2}^{p} P_{2}}{g_{s}^{p} \lambda P_{s}+N_{0}}\right)+\tau\left(\frac{g_{s}^{s} \lambda P_{s}}{g_{2}^{s} P_{2}+N_{0}}\right) \\
& -\left[\tau\left(\frac{g_{1}^{p} P_{1}}{g_{s}^{p} \lambda P_{s}+g_{2}^{p} P_{2}+N_{0}}\right)-\tau\left(\frac{g_{1}^{s} P_{1}}{g_{s}^{s} \lambda P_{s}+g_{2}^{s} P_{2}+N_{0}}\right)\right]^{+} \\
& +\min \left\{\tau\left(\frac{g_{s}^{p} \bar{\lambda} P_{s}}{g_{s}^{p} \lambda P_{s}+g_{1}^{p} P_{1}+g_{2}^{p} P_{2}+N_{0}}\right),\right. \\
& \tau\left(\frac{g_{s}^{s} \bar{\lambda} P_{s}}{g_{s}^{s} \lambda P_{s}+g_{1}^{s} P_{1}+g_{2}^{s} P_{2}+N_{0}}\right)+\left[\tau\left(\frac{g_{1}^{s} P_{1}}{g_{s}^{s} \lambda P_{s}+g_{2}^{s} P_{2}+N_{0}}\right)\right. \\
& \left.\left.-\tau\left(\frac{g_{1}^{p} P_{1}}{g_{s}^{p} \lambda P_{s}+g_{2}^{p} P_{2}+N_{0}}\right)\right]^{+}, \tau\left(\frac{g_{s}^{s} \bar{\lambda} P_{s}}{g_{s}^{s} \lambda P_{s}+g_{2}^{s} P_{2}+N_{0}}\right)\right\} .
\end{aligned}
$$

- If $\tau\left(\frac{g_{1}^{p} P_{1}}{g_{s}^{p} \lambda P_{s}+g_{2}^{p} P_{2}+N_{0}}\right) \leq \tau\left(\frac{g_{1}^{s} P_{1}}{g_{s}^{s} \lambda P_{s}+g_{2}^{s} P_{2}+N_{0}}\right)$ 


$$
\begin{aligned}
& \rho_{s 2}^{r}=2 \tau\left(\frac{g_{1}^{p} P_{1}+g_{2}^{p} P_{2}}{g_{s}^{p} \lambda P_{s}+N_{0}}\right)+\tau\left(\frac{g_{s}^{s} \lambda P_{s}}{g_{2}^{s} P_{2}+N_{0}}\right)+\min \\
& \overbrace{\tau\left(\frac{g_{s}^{p} \bar{\lambda} P_{s}}{g_{s}^{p} \lambda P_{s}+g_{1}^{p} P_{1}+g_{2}^{p} P_{2}+N_{0}}\right)}^{\mu_{5}}, \overbrace{\tau\left(\frac{g_{s}^{s} \bar{\lambda} P_{s}}{g_{s}^{s} \lambda P_{s}+g_{2}^{s} P_{2}+N_{0}}\right)}^{\mu_{6}}, \\
& \overbrace{\tau\left(\frac{g_{s}^{s} \bar{\lambda} P_{s}+g_{1}^{s} P_{1}}{g_{s}^{s} \lambda P_{s}+g_{2}^{s} P_{2}+N_{0}}\right)-\tau\left(\frac{g_{1}^{p} P_{1}}{g_{s}^{p} \lambda P_{s}+g_{2}^{p} P_{2}+N_{0}}\right)}^{\mu_{7}}\} \text {. }
\end{aligned}
$$

When $\mu_{5}=\min \left\{\mu_{5}, \mu_{6}, \mu_{7}\right\}$ in (121) we have

$$
\begin{aligned}
\rho_{s 2}^{r}= & \tau\left(\frac{g_{s}^{p} \bar{\lambda} P_{s}+g_{1}^{p} P_{1}+g_{2} p P_{2}}{g_{s}^{p} \lambda P_{s}+N_{0}}\right)+\tau\left(\frac{g_{s}^{s} \lambda P_{s}}{g_{2}^{s} P_{2}+N_{0}}\right) \\
& +\tau\left(\frac{g_{1}^{p} P_{1}+g_{2}^{p} P_{2}}{g_{s}^{p} \lambda P_{s}+N_{0}}\right) .
\end{aligned}
$$

Note that the third term in (122) is decreasing with $\lambda$, and the first derivative of the first two terms with respect to $\lambda$ is given by

$$
\begin{aligned}
& -\frac{0.5 P_{s}\left(g_{s}^{p} g_{2}^{s} P_{2}+g_{s}^{p} N_{0}-g_{s}^{s} N_{0}\right)}{\ln 2\left(g_{s}^{p} \lambda P_{s}+N_{0}\right)\left(g_{s}^{s} \lambda P_{s}+g_{2}^{s} P_{2}+N_{0}\right)} \\
& -\frac{0.5 g_{s}^{p} P_{s}\left(g_{2}^{p} P_{2}+g_{1}^{p} P_{1}\right)}{\ln 2\left(g_{s}^{p} \lambda P_{s}+N_{0}\right)\left(g_{s}^{p} \lambda P_{s}+g_{1}^{p} P_{1}+g_{2}^{p} P_{2}+N_{0}\right)} .
\end{aligned}
$$

Since inequality (99) is satisfied for user 1 , then the derivative is negative and consequently $\rho_{s 2}^{r}$ is decreasing with $\lambda$.

When $\mu_{6}=\min \left\{\mu_{5}, \mu_{6}, \mu_{7}\right\}$ in (121), we have

$$
\rho_{s 2}^{r}=2 \tau\left(\frac{g_{1}^{p} P_{1}+g_{2}^{p} P_{2}}{g_{s}^{p} \lambda P_{s}+N_{0}}\right)+\tau\left(\frac{g_{s}^{s} P_{s}}{g_{2}^{s} P_{2}+N_{0}}\right),
$$

i.e., $\rho_{s 2}^{r}$ is decreasing with $\lambda$.

When $\mu_{7}=\min \left\{\mu_{5}, \mu_{6}, \mu_{7}\right\}$ in (121), we have

$$
\begin{aligned}
\rho_{s 2}^{r}= & 2 \tau\left(\frac{g_{1}^{p} P_{1}+g_{2}^{p} P_{2}}{g_{s}^{p} \lambda P_{s}+N_{0}}\right)-\tau\left(\frac{g_{1}^{p} P_{1}}{g_{s}^{p} \lambda P_{s}+g_{2}^{p} P_{2}+N_{0}}\right) \\
& +\tau\left(\frac{g_{s}^{s} P_{s}+g_{1}^{s} P_{1}}{g_{2}^{s} P_{2}+N_{0}}\right) .
\end{aligned}
$$

Hence, $\rho_{s 2}^{r}$ is decreasing with $\lambda$.

- If $\tau\left(\frac{g_{1}^{s} P_{1}}{g_{s}^{s} \lambda P_{s}+g_{2}^{g} P_{2}+N_{0}}\right) \leq \tau\left(\frac{g_{1}^{p} P_{1}}{g_{s}^{p} \lambda P_{s}+g_{2}^{p} P_{2}+N_{0}}\right)$

$$
\begin{aligned}
& \rho_{s 2}^{r}=2 \tau\left(\frac{g_{1}^{p} P_{1}+g_{2}^{p} P_{2}}{g_{s}^{p} \lambda P_{s}+N_{0}}\right)-\tau\left(\frac{g_{1}^{p} P_{1}}{g_{s}^{p} \lambda P_{s}+g_{2}^{p} P_{2}+N_{0}}\right) \\
& +\min \{\overbrace{\tau\left(\frac{g_{s}^{p} \bar{\lambda} P_{s}}{\mu_{s}^{p} \lambda P_{s}+g_{1}^{p} P_{1}+g_{2}^{p} P_{2}+N_{0}}\right)}^{\mu_{5}}, \\
& \overbrace{\tau\left(\frac{g_{s}^{s} \bar{\lambda} P_{s}}{g_{s}^{s} \lambda P_{s}+g_{1}^{s} P_{1}+g_{2}^{s} P_{2}+N_{0}}\right)}^{\mu_{8}}\}+\tau\left(\frac{g_{s}^{s} \lambda P_{s}+g_{1}^{s} P_{1}}{g_{2}^{s} P_{2}+N_{0}}\right)
\end{aligned}
$$

When $\mu_{5}=\min \left\{\mu_{5}, \mu_{8}\right\}$ in (123), then

$$
\begin{aligned}
\rho_{s 2}^{r}= & \tau\left(\frac{g_{1}^{p} P_{1}+g_{2}^{p} P_{2}}{g_{s}^{p} \lambda P_{s}+N_{0}}\right)-\tau\left(\frac{g_{1}^{p} P_{1}}{g_{s}^{p} \lambda P_{s}+g_{2}^{p} P_{2}+N_{0}}\right) \\
& +\tau\left(\frac{g_{1}^{p} P_{1}+g_{2}^{p} P_{2}}{g_{s}^{p} \lambda P_{s}+N_{0}}\right)+\tau\left(\frac{g_{s}^{s} \lambda P_{s}+g_{1}^{s} P_{1}}{g_{2}^{s} P_{2}+N_{0}}\right) \\
& +\tau\left(\frac{g_{s}^{p} \bar{\lambda} P_{s}}{g_{s}^{p} \lambda P_{s}+g_{1}^{p} P_{1}+g_{2}^{p} P_{2}+N_{0}}\right) .
\end{aligned}
$$

For all values of $0 \leq \lambda \leq 1$, the difference between the first two terms in (124) is always positive and decreasing as $\lambda$ increases. To see this, we can write such difference as $I\left(Y_{p} ; X_{1} X_{2} \mid W\right)-I\left(Y_{p}, X 1 \mid W\right)=I\left(Y_{p} ; X 2 \mid W\right)=$ $\tau\left(\frac{g 2^{2} P_{2}}{\lambda g_{s}^{p} P_{s}}\right)$. The first derivative of the last three terms in (124) with respect to $\lambda$ is given by

$$
\begin{aligned}
& -\frac{0.5 P_{s}\left(g_{s}^{p} g_{2}^{s} P_{2}+g_{s}^{p} g_{1}^{s} P_{1}+g_{s}^{p} N_{0}-g_{s}^{s} N_{0}\right)}{\ln 2\left(g_{s}^{p} \lambda P_{s}+N_{0}\right)\left(g_{s}^{s} \lambda P_{s}+g_{2}^{s} P_{2}+g_{1}^{s} P_{1}+N_{0}\right)} \\
& \leq 0 \text { from (99). }
\end{aligned}
$$

Therefore, $\rho_{s 2}^{r}$ is decreasing with $\lambda$.

When $\mu_{8}=\min \left\{\mu_{5}, \mu_{8}\right\}$ in (123), then

$$
\begin{aligned}
\rho_{s 2}^{r}= & 2 \tau\left(\frac{g_{1}^{p} P_{1}+g_{2}^{p} P_{2}}{g_{s}^{p} \lambda P_{s}+N_{0}}\right)-\tau\left(\frac{g_{1}^{p} P_{1}}{g_{s}^{p} \lambda P_{s}+g_{2}^{p} P_{2}+N_{0}}\right) \\
& +\tau\left(\frac{g_{s}^{s} P_{s}+g_{1}^{s} P_{1}}{g_{2}^{s} P_{2}+N_{0}}\right) .
\end{aligned}
$$

In the above formula, the difference between the first two terms is always positive and decreasing as $\lambda$ increases. 
The third term does not depend on $\lambda$. Hence, $\rho_{s 2}^{r}$ is decreasing with $\lambda$.

$$
\begin{aligned}
& \text { At } \sigma_{p}^{*}=\tau\left(\frac{g_{1}^{s} P_{1}}{g_{2}^{s} P_{2}+N_{0}}\right) \leq \tau\left(\frac{g_{1}^{p} P_{1}}{g_{s}^{p} \lambda P_{s}+g_{2}^{p} P_{2}+N_{0}}\right) \\
& \rho_{s 2}^{r}=2 \tau\left(\frac{g_{2}^{p} P_{2}}{g_{s}^{p} \lambda P_{s}+N_{0}}\right)+\tau\left(\frac{g_{1}^{s} P_{1}}{g_{2}^{s} P_{2}+N_{0}}\right)+\tau\left(\frac{g_{s}^{s} \lambda P_{s}+g_{1}^{s} P_{1}}{g_{2}^{s} P_{2}+N_{0}}\right) \\
& +\min \{\overbrace{\tau\left(\frac{g_{s}^{s} \bar{\lambda} P_{s}}{g_{s}^{s} \lambda P_{s}+g_{1}^{s} P_{1}+g_{2}^{s} P_{2}+N_{0}}\right)}^{\mu_{8}}, \\
& \overbrace{\tau\left(\frac{g_{s}^{p} \bar{\lambda} P_{s}}{g_{s}^{p} \lambda P_{s}+g_{2}^{p} P_{2}+N_{0}}\right)}^{\mu_{9}} \\
& \overbrace{\tau\left(\frac{g_{s}^{p} \bar{\lambda} P_{s}+g_{1}^{p} P_{1}}{g_{s}^{p} \lambda P_{s}+g_{2}^{p} P_{2}+N_{0}}\right)-\tau\left(\frac{g_{1}^{s} P_{1}}{g_{2}^{s} P_{2}+N_{0}}\right)}^{\mu_{10}}\} .
\end{aligned}
$$

When $\mu_{8}=\min \left\{\mu_{8}, \mu_{9}, \mu_{10}\right\}$ in (125), we have

$$
\begin{aligned}
\rho_{s 2}^{r}= & 2 \tau\left(\frac{g_{2}^{p} P_{2}}{g_{s}^{p} \lambda P_{s}+N_{0}}\right)+\tau\left(\frac{g_{1}^{s} P_{1}}{g_{2}^{s} P_{2}+N_{0}}\right) \\
& +\tau\left(\frac{g_{s}^{s} P_{s}+g_{1}^{s} P_{1}}{g_{2}^{s} P_{2}+N_{0}}\right) .
\end{aligned}
$$

That is, $\rho_{s 2}^{r}$ is decreasing with $\lambda$.

When $\mu_{9}=\min \left\{\mu_{8}, \mu_{9}, \mu_{10}\right\}$ in (125), we have

$$
\begin{aligned}
\rho_{s 2}^{r}= & \tau\left(\frac{g_{2}^{p} P_{2}}{g_{s}^{p} \lambda P_{s}+N_{0}}\right)+\tau\left(\frac{g_{1}^{s} P_{1}}{g_{2}^{s} P_{2}+N_{0}}\right) \\
& +\tau\left(\frac{g_{s}^{s} \lambda P_{s}+g_{1}^{s} P_{1}}{g_{2}^{s} P_{2}+N_{0}}\right)+\tau\left(\frac{g_{s}^{p} \bar{\lambda} P_{s}+g_{2}^{p} P_{2}}{g_{s}^{p} \lambda P_{s}+N_{0}}\right) .
\end{aligned}
$$

The first term in (126) is decreasing with $\lambda$ for all values of $\lambda$. The first derivative of the other terms with respect to $\lambda$ is given by

$$
\begin{aligned}
& -\frac{0.5 P_{s}\left(g_{s}^{p} g_{s}^{s} P_{2}+g_{s}^{p} g_{1}^{s} P_{1}+g_{s}^{p} N_{0}-g_{s}^{s} N_{0}\right)}{\ln 2\left(g_{s}^{p} \lambda P_{s}+N_{0}\right)\left(g_{s}^{s} \lambda P_{s}+g_{2}^{s} P_{2}+g_{1}^{s} P_{1}+N_{0}\right)} \\
& \leq 0 \text { from (99). }
\end{aligned}
$$

Hence, $\rho_{s 2}^{r}$ is decreasing with $\lambda$.

When $\mu_{10}=\min \left\{\mu_{8}, \mu_{9}, \mu_{10}\right\}$ in (125), we have

$$
\begin{aligned}
\rho_{s 2}^{r}= & \tau\left(\frac{g_{2}^{p} P_{2}}{g_{s}^{p} \lambda P_{s}+N_{0}}\right)+\tau\left(\frac{g_{s}^{p} \bar{\lambda} P_{s}+g_{1}^{p} P_{1}+g_{2}^{p} P_{2}}{g_{s}^{p} \lambda P_{s}+N_{0}}\right) \\
& +\tau\left(\frac{g_{s}^{s} \lambda P_{s}+g_{1}^{s} P_{1}}{g_{2}^{s} P_{2}+N_{0}}\right) .
\end{aligned}
$$

The first term in (127) is decreasing with $\lambda$, and the first derivative of the other three terms with respect to $\lambda$ is given by

$$
\begin{aligned}
& -\frac{0.5 P_{s}\left(g_{s}^{p} g_{2}^{s} P_{2}+g_{s}^{p} g_{1}^{s} P_{1}+g_{s}^{p} N_{0}-g_{s}^{s} N_{0}\right)}{\ln 2\left(g_{s}^{p} \lambda P_{s}+N_{0}\right)\left(g_{s}^{s} \lambda P_{s}+g_{1}^{s} P_{1}+g_{2}^{s} P_{2}+N_{0}\right)} \\
& \leq 0 \text { from (99). }
\end{aligned}
$$

Thus, $\rho_{s 2}^{r}$ is decreasing with $\lambda$.

$2 R_{s}^{r}+R_{p}^{r}=\rho_{2 p}^{r}$

From (99),

$$
\begin{aligned}
& \sigma_{s}^{*}=\tau\left(\frac{g_{s}^{s} \bar{\lambda} P_{s}}{g_{s}^{s} \lambda P_{s}+g_{2}^{s} P_{2}+N_{0}}\right) . \\
& \rho_{2 p}^{r}=2 \tau\left(\frac{g_{s}^{s} P_{s}}{g_{2}^{s} P_{2}+N_{0}}\right)+\tau\left(\frac{g_{2}^{p} P_{2}}{g_{s}^{p} \lambda P_{s}+N_{0}}\right) \\
& -\left[\tau\left(\frac{g_{s}^{s} \bar{\lambda} P_{s}}{g_{s}^{s} \lambda P_{s}+g_{2}^{s} P_{2}+N_{0}}\right)-\tau\left(\frac{g_{s}^{p} \bar{\lambda} P_{s}}{g_{s}^{p} \lambda P_{s}+g_{2}^{p} P_{2}+N_{0}}\right)\right]^{+} \\
& +\min \left\{\tau\left(\frac{g_{1}^{s} P_{1}}{g_{s}^{s} P_{s}+g_{2}^{s} P_{2}+N_{0}}\right), \tau\left(\frac{g_{1}^{p} P_{1}}{g_{s}^{p} P_{s}+g_{2}^{p} P_{2}+N_{0}}\right)\right. \\
& +\left[\tau\left(\frac{g_{s}^{p} \bar{\lambda} P_{s}}{g_{s}^{p} \lambda P_{s}+g_{2}^{p} P_{2}+N_{0}}\right)-\tau\left(\frac{g_{s}^{s} \bar{\lambda} P_{s}}{g_{s}^{s} \lambda P_{s}+g_{2}^{s} P_{2}+N_{0}}\right)\right]^{+}, \\
& \left.\tau\left(\frac{g_{1}^{p} P_{1}}{g_{s}^{p} \lambda P_{s}+g_{2}^{p} P_{2}+N_{0}}\right)\right\} \text {. } \\
& \text { If } \tau\left(\frac{g_{s}^{s} \bar{\lambda} P_{s}}{g_{s}^{s} \lambda P_{s}+g_{2}^{s} P_{2}+N_{0}}\right) \leq \tau\left(\frac{g_{s}^{p} \bar{\lambda} P_{s}}{g_{s}^{p} \lambda P_{s}+g_{2}^{p} P_{2}+N_{0}}\right) \\
& \rho_{2 p}^{r}=2 \tau\left(\frac{g_{s}^{s} P_{s}}{g_{2}^{s} P_{2}+N_{0}}\right)+\tau\left(\frac{g_{2}^{p} P_{2}}{g_{s}^{p} \lambda P_{s}+N_{0}}\right) \\
& +\min \{\overbrace{\tau\left(\frac{g_{1}^{s} P_{1}}{g_{s}^{s} \lambda P_{s}+g_{2}^{s} P_{2}+N_{0}}\right)}^{\mu_{11}}, \overbrace{\tau\left(\frac{g_{1}^{p} P_{1}}{g_{s}^{p} \lambda P_{s}+g_{2}^{p} P_{2}+N_{0}}\right)}^{\mu_{12}}, \\
& \overbrace{\tau\left(\frac{g_{1}^{p} P_{1}+g_{s}^{p} \bar{\lambda} P_{s}}{g_{s}^{p} \lambda P_{s}+g_{2}^{p} P_{2}+N_{0}}\right)-\tau\left(\frac{g_{s}^{s} \bar{\lambda} P_{s}}{g_{s}^{s} \lambda P_{s}+g_{2}^{s} P_{2}+N_{0}}\right)}^{\mu_{13}}) .
\end{aligned}
$$

- When $\mu_{11}=\min \left\{\mu_{11}, \mu_{12}, \mu_{13}\right\}$ in (128), then

$$
\begin{aligned}
\rho_{2 p}^{r}= & 2 \tau\left(\frac{g_{s}^{s} P_{s}}{g_{2}^{s} P_{2}+N_{0}}\right)+\tau\left(\frac{g_{2}^{p} P_{2}}{g_{s}^{p} \lambda P_{s}+N_{0}}\right) \\
& \tau\left(\frac{g_{1}^{s} P_{1}}{g_{s}^{s} \lambda P_{s}+g_{2}^{s} P_{2}+N_{0}}\right) .
\end{aligned}
$$

It is clear that $\rho_{2 p}^{r}$ is decreasing with $\lambda$. 
- When $\mu_{12}=\min \left\{\mu_{11}, \mu_{12}, \mu_{13}\right\}$ in (128), then

$$
\begin{aligned}
\rho_{2 p}^{r}= & 2 \tau\left(\frac{g_{s}^{s} P_{s}}{g_{2}^{s} P_{2}+N_{0}}\right)+\tau\left(\frac{g_{2}^{p} P_{2}}{g_{s}^{p} \lambda P_{s}+N_{0}}\right) \\
& +\tau\left(\frac{g_{1}^{p} P_{1}}{g_{s}^{p} \lambda P_{s}+g_{2}^{p} P_{2}+N_{0}}\right) .
\end{aligned}
$$

It is also clear that $\rho_{2 p}^{r}$ is decreasing with $\lambda$.

- When $\mu_{13}=\min \left\{\mu_{11}, \mu_{12}, \mu_{13}\right\}$ in (128), then

$$
\begin{aligned}
\rho_{2 p}^{r}= & 2 \tau\left(\frac{g_{s}^{s} P_{s}}{g_{2}^{s} P_{2}+N_{0}}\right)+\tau\left(\frac{g_{1}^{p} P_{1}}{g_{s}^{p} P_{s}+g_{2}^{p} P_{2}+N_{0}}\right) \\
& +\tau\left(\frac{g_{s}^{p} \bar{\lambda} P_{s}+g_{2}^{p} P_{2}}{g_{s}^{p} \lambda P_{s}+N_{0}}\right)-\tau\left(\frac{g_{s}^{s} \bar{\lambda} P_{s}}{g_{s}^{s} \lambda P_{s}+g_{2}^{s} P_{2}+N_{0}}\right) . \\
\frac{\partial \rho_{2 p}^{r}}{\partial \lambda}= & -\frac{0.5 P_{s}\left(g_{s}^{p} g_{2}^{s} P_{2}+g_{s}^{p} N_{0}-g_{s}^{s} N_{0}\right)}{\ln 2\left(g_{s}^{p} \lambda P_{s}+N_{0}\right)\left(g_{s}^{s} \lambda P_{s}+g_{2}^{s} P_{2}+N_{0}\right)} \\
& \leq 0 \quad \text { from (99). }
\end{aligned}
$$

Thus, $\rho_{2 p}^{r}$ is decreasing with $\lambda$.

$$
\begin{aligned}
& \text { If } \boldsymbol{\tau}\left(\frac{\boldsymbol{g}_{s}^{p} \bar{\lambda} P_{s}}{g_{s}^{p} \lambda P_{s}+g_{2}^{p} P_{2}+N_{0}}\right) \leq \tau\left(\frac{g_{s}^{s} \bar{\lambda} P_{s}}{g_{s}^{s} \lambda P_{s}+g_{2}^{s} P_{2}+N_{0}}\right) \\
& \rho_{2 p}^{r}=2 \tau\left(\frac{g_{s}^{s} P_{s}}{g_{2}^{s} P_{2}+N_{0}}\right)+\tau\left(\frac{g_{s}^{p} \bar{\lambda} P_{s}+g_{2}^{p} P_{2}}{g_{s}^{p} \lambda P_{s}+N_{0}}\right) \\
& \quad-\tau\left(\frac{g_{s}^{s} \bar{\lambda} P_{s}}{g_{s}^{s} \lambda P_{s}+g_{2}^{s} P_{2}+N_{0}}\right)+\min \left\{\tau\left(\frac{g_{1}^{s} P_{1}}{g_{s}^{s} P_{s}+g_{2}^{s} P_{2}+N_{0}}\right),\right. \\
& \left.\tau\left(\frac{g_{1}^{p} P_{1}}{g_{s}^{p} P_{s}+g_{2}^{p} P_{2}+N_{0}}\right)\right\} . \\
& \frac{\partial \rho_{2 p}^{r}}{\partial \lambda}=-\frac{0.5 P_{s}\left(g_{s}^{p} g_{2}^{s} P_{2}+g_{s}^{p} N_{0}-g_{s}^{s} N_{0}\right)}{\ln 2\left(g_{s}^{p} \lambda P_{s}+N_{0}\right)\left(g_{s}^{s} \lambda P_{s}+g_{2}^{s} P_{2}+N_{0}\right)} \\
& \leq 0 \quad \text { from (99). }
\end{aligned}
$$

Therefore, $\rho_{2 p}^{r}$ is decreasing with $\lambda$.

Thus, since we showed that if (99) is satisfied, assuming that the secondary receiver can decode the signal of primary user 1 , then $\rho_{p}^{r}, \rho_{s p}^{r}, \rho_{s 2}^{r}$, and $\rho_{2 p}^{r}$ decrease with $\lambda$, whereas $\rho_{s}^{r}$ does not depend on $\lambda$; hence, $\mathcal{R}_{1}^{r}(Z)$ at $\lambda=0$ coincides on $\mathcal{R}_{1 g}^{r}$. And for any $\lambda_{1}$ and $\lambda_{2}$ such that $\lambda_{1}>\lambda_{2}$, $\mathcal{R}_{1}^{r}(Z)$ at $\lambda_{1}$ is a subset of $\mathcal{R}_{1}^{r}(Z)$ at $\lambda_{2}$.

\section{Necessity part}

In this part of the proof, we show that if condition (99) is not satisfied, then $\mathcal{R}_{1 g}^{r}$ does not coincide on any $\mathcal{R}_{1}^{r}(Z)$ for all values of $\lambda$. So, assume that (99) is not satisfied, i.e.,

$$
N_{0} g_{s}^{s}>g_{s}^{p} g_{2}^{s} P_{2}+g_{s}^{p} N_{0} .
$$

By referring to Figure 4 , the effect of $\lambda$ on $\mathcal{R}_{1}^{r}(Z)$ at points $A$ and $F$ is determined as follows.

\section{At point $A$}

$$
\begin{aligned}
R_{p}^{r A}= & \tau\left(\frac{g_{2}^{p} P_{2}}{g_{s}^{p} \lambda P_{s}+N_{0}}\right)+\min \left\{\tau\left(\frac{g_{1}^{s} P_{1}}{g_{2}^{s} P_{2}+N_{0}}\right),\right. \\
& \left.\tau\left(\frac{g_{1}^{p} P_{1}}{g_{s}^{p} \lambda P_{s}+g_{2}^{p} P_{2}+N_{0}}\right)\right\} .
\end{aligned}
$$

It is clear that $R_{p}^{r A}$ is decreasing with $\lambda$.

\section{At point $F$}

$$
\begin{aligned}
R_{s}^{r F}= & \tau\left(\frac{g_{s}^{s} \lambda P_{s}}{g_{2}^{s} P_{2}+N_{0}}\right)+\tau\left(\frac{g_{s}^{p} \bar{\lambda} P_{s}}{g_{s}^{p} \lambda P_{s}+N_{0}}\right) . \\
\frac{\partial R_{s}^{r F}}{\partial \lambda} & =\frac{0.5 P_{s}\left(g_{s}^{s} N_{0}-\left(g_{s}^{p} g_{s}^{s} P_{2}+g_{s}^{p} N_{0}\right)\right)}{\ln 2\left(g_{s}^{p} \lambda P_{s}+N_{0}\right)\left(g_{s}^{s} \lambda P_{s}+g_{2}^{s} P_{2}+N_{0}\right)} \\
& >0 \text { from (129). }
\end{aligned}
$$

Consequently, $R_{s}^{r F}$ is increasing with $\lambda$.

So, for any two different values of $\lambda$, the corresponding rate regions $\mathcal{R}_{1}^{r}(Z)$ do not include one another; thus, $\mathcal{R}_{1 g}^{r}$ does not coincide on $\mathcal{R}_{1}^{r}(Z)$ at any value of $\lambda$.

\section{Competing interests}

The authors declare that they have no competing interests.

\section{Author details}

${ }^{1}$ Department of Electrical and Computer Engineering, Rice University, Houston, TX 77025, USA. ${ }^{2}$ Department of Electronics and Communications, Faculty of Engineering, Cairo University, Cairo 12316, Egypt. ${ }^{3}$ Wireless Intelligent Networks Center (WINC), Nile University, Cairo 12677, Egypt.

\section{Received: 11 November 2013 Accepted: 27 October 2014}

Published: 28 November 2014

\section{References}

1. III Mitola J, Cognitive radio: an integrated agent architecture for software defined radio. Doctor of Technology Dissertation, Royal Institute of Technology (KTH), Sweden, May, 2000

2. IF Akyildiz, W-Y Lee, MC Vuran, S Mohanty, NeXt generation/dynamic spectrum access/cognitive radio wireless networks: a survey. Comput. Netw. J. (Elsevier). 50(13), 2127-2159 (2006)

3. SA Jafar, S Srinivasa, I Maric, A Goldsmith, Breaking spectrum gridlock with cognitive radios: an information theoretic perspective. Proc. IEEE. 97(5), 894-914 (2009)

4. N Devroye, P Mitran, V Tarokh, Achievable rates in cognitive radio channels. IEEE Trans. Inform. Theory. 52, 1813-1827 (2006)

5. TS Han, K Kobayashi, A new achievable rate region for the interference channel. IEEE. Trans. Info. Theory. 27, 49-60 (1981)

6. I Maric, RD Yates, G Kramer, Capacity of interference channels with partial transmitter cooperation. IEEE. Trans. Info. Theory. 53(10), 3536-3548 (2007)

7. I Maric, A Goldsmith, G Kramer, S Shamai (Shitz), On the capacity of interference channels with one cooperating transmitter. European Trans. Telecomm. 19(4), 405-420 (2008)

8. Y Pang, MK Varanasi, in 201351 st Annual Allerton Conference on Communication, Control, and Computing (Allerton). Bounds on the capacity region of a class of multiple access interference channels (Monticello, IL, 2-4 October 2013), pp. 599-606

9. R Fritschek, G Wunder, Enabling the multi-user generalized degrees of freedom in the Gaussian cellular channel. Available online at: http://arxiv. org/abs/1408.5072, Aug. 2014

10. A Chaaban, A Sezgin, B Bandemer, A Paulraj, in Proceedings of MACOM'12. On Gaussian multiple access channels with interference: achievable rates and upper bounds (Trento, Italy, 12-13 September 2011) 
11. J Tadrous, A Sultan, M Nafie, in IEEE 17th International Conference on Telecommunications. An achievable rate region for a primary network shared by a secondary link (Doha, Qatar, April 2010), pp. 77-82

12. Y Xing, CN Mathur, MA Haleem, R Chandramouli, KP Subbalakshmi, Dynamic spectrum access with QoS and interference temperature constraints. IEEE Trans. Mobile Comp. 6(4), 423-433 (2007)

13. L Le, E Hossain, Resource allocation for spectrum underlay in cognitive radio networks. IEEE Trans. Wireless Commun. 7(12), 5306-5315 (2008)

14. DI Kim, L Le, E Hossain, Joint rate and power allocation for cognitive radios in dynamic spectrum access environment. IEEE Trans. Wireless Commun. 7(12), 5517-5527 (2008)

15. J Tadrous, A Sultan, M Nafie, A El-Keyi, in 2010 IEEE Global Telecommunications Conference (GLOBECOM 2010). Power control for constrained throughput maximization in spectrum shared networks (Miami, FL, 6-10 December 2010), pp. 1-6

16. J Tadrous, A Sultan, M Nafie, Admission and power control for spectrum sharing cognitive radio networks. IEEE Trans. Wireless Commun. 10(6), 1945-1955 (2011)

17. P Popovski, H Yomo, K Nishimori, R Taranto Di, R Prasad, in IEEE International Symposium on New Frontiers in Dynamic Spectrum Access Networks. Opportunistic interference cancellation in cognitive radio systems, (April 2007), pp. 472-475

18. T Cover, J Thomas, Elements of Information Theory. (Wiley, 2006)

19. HF Chong, M Motani, HK Garg, H El Gamal, On the Han-Kobayashi region for the interference channel. IEEE Trans. Inf. Theory. 54(7), 3188-3195 (2008)

doi:10.1186/1687-1499-2014-203

Cite this article as: Tadrous and Nafie: On the achievable rates of a secondary link coexisting with a primary multiple access network. EURASIP Journal on Wireless Communications and Networking 2014 2014:203.

\section{Submit your manuscript to a SpringerOpen ${ }^{\mathcal{O}}$ journal and benefit from:}

- Convenient online submission

- Rigorous peer review

- Immediate publication on acceptance

- Open access: articles freely available online

- High visibility within the field

- Retaining the copyright to your article

Submit your next manuscript at $\boldsymbol{\wedge}$ springeropen.com 